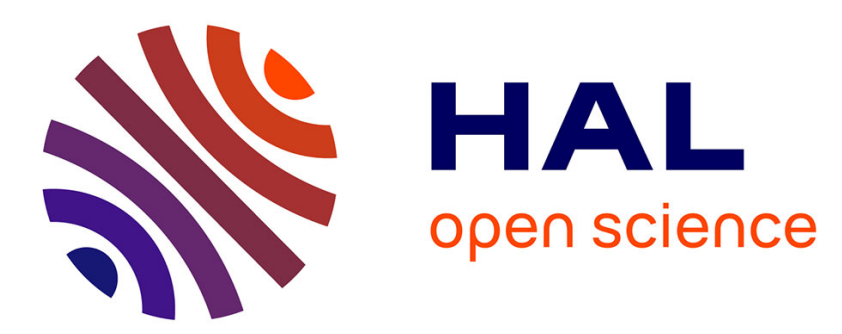

\title{
Inertial dynamics of air bubbles crossing a horizontal fluid-fluid interface
}

\author{
Romain Bonhomme, Jacques Magnaudet, Fabien Duval, Bruno Piar
}

\section{To cite this version:}

Romain Bonhomme, Jacques Magnaudet, Fabien Duval, Bruno Piar. Inertial dynamics of air bubbles crossing a horizontal fluid-fluid interface. Journal of Fluid Mechanics, 2012, vol. 707, pp. 405-443. 10.1017/jfm.2012.288 . hal-00908876

\section{HAL Id: hal-00908876 https://hal.science/hal-00908876}

Submitted on 25 Nov 2013

HAL is a multi-disciplinary open access archive for the deposit and dissemination of scientific research documents, whether they are published or not. The documents may come from teaching and research institutions in France or abroad, or from public or private research centers.
L'archive ouverte pluridisciplinaire HAL, est destinée au dépôt et à la diffusion de documents scientifiques de niveau recherche, publiés ou non, émanant des établissements d'enseignement et de recherche français ou étrangers, des laboratoires publics ou privés. 


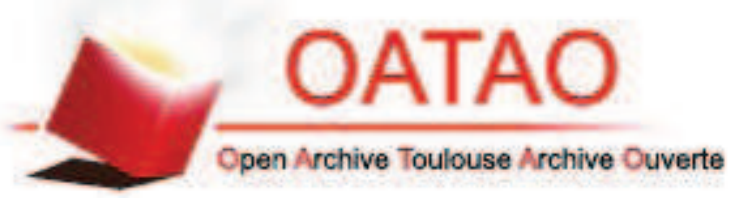

Open Archive TOULOUSE Archive Ouverte (OATAO)

OATAO is an open access repository that collects the work of Toulouse researchers and makes it freely available over the web where possible.

This is an author-deposited version published in : http://oatao.univ-toulouse.fr/ Eprints ID : 10245

To link to this article : DOI: $10.1017 / \mathrm{jfm} .2012 .288$

URL : http://dx.doi.org/10.1017/jfm.2012.288

To cite this version : Bonhomme, Romain and Magnaudet, Jacques and Duval, Fabien and Piar, Bruno Inertial dynamics of air bubbles crossing a horizontal fluid-fluid interface. (2012) Journal of Fluid Mechanics, vol. 707. pp. 405-443. ISSN 0022-1120

Any correspondance concerning this service should be sent to the repository administrator: staff-oatao@ listes-diff.inp-toulouse.fr 


\title{
Inertial dynamics of air bubbles crossing a horizontal fluid-fluid interface
}

\author{
Romain Bonhomme $e^{1,2,3}$, Jacques Magnaudet ${ }^{1,2} \dagger$, Fabien Duval $^{3}$ \\ and Bruno Piar ${ }^{3}$ \\ ${ }^{1}$ Université de Toulouse; INPT, UPS; IMFT (Institut de Mécanique des Fluides de Toulouse); \\ Allée Camille Soula, F-31400 Toulouse, France \\ 2 CNRS; IMFT; F-31400 Toulouse, France \\ ${ }^{3}$ Institut de Radioprotection et de Sûreté Nucléaire, BP 3, 13115 St Paul lez Durance CEDEX, France
}

The dynamics of isolated air bubbles crossing the horizontal interface separating two Newtonian immiscible liquids initially at rest are studied both experimentally and computationally. High-speed video imaging is used to obtain a detailed evolution of the various interfaces involved in the system. The size of the bubbles and the viscosity contrast between the two liquids are varied by more than one and four orders of magnitude, respectively, making it possible to obtain bubble shapes ranging from spherical to toroidal. A variety of flow regimes is observed, including that of small bubbles remaining trapped at the fluid-fluid interface in a film-drainage configuration. In most cases, the bubble succeeds in crossing the interface without being stopped near its undisturbed position and, during a certain period of time, tows a significant column of lower fluid which sometimes exhibits a complex dynamics as it lengthens in the upper fluid. Direct numerical simulations of several selected experimental situations are performed with a code employing a volume-of-fluid type formulation of the incompressible Navier-Stokes equations. Comparisons between experimental and numerical results confirm the reliability of the computational approach in most situations but also points out the need for improvements to capture some subtle but important physical processes, most notably those related to film drainage. Influence of the physical parameters highlighted by experiments and computations, especially that of the density and viscosity contrasts between the two fluids and of the various interfacial tensions, is discussed and analysed in the light of simple models and available theories.

Key words: capillary flows, drops and bubbles, multiphase flows

\section{Introduction}

Buoyancy-driven drops and bubbles crossing horizontal liquid-liquid interfaces are encountered in a variety of engineering situations such as liquid-liquid extraction, emulsification or iron processing. For instance, the ladle stirring technique widely employed in iron processing makes use of nitrogen bubbles injected at the bottom of the device to stir and mix the liquid metal and remove impurities (Poggi, Minto \& Davenport 1969; Kobayashi 1993). Some nuclear accident scenarios also consider the 
situation where the concrete slab below the reactor is ablated by the fuel-containing material (corium) and releases gas bubbles. In both cases, due to compositional differences, the fluid has a two-layer structure with an upper layer made of slag in the former case and predominantly of oxides in the latter. Similar configurations are found in geophysical problems where fluids undergo natural discontinuous density and/or viscosity stratification, such as the ascent of plumes through the Earth's mantle (Manga, Stone \& O'Connell 1993). In microfluidics, two-layer fluid systems may be used to coat paramagnetic drops or particles with a shell made of the lower fluid, buoyancy then being replaced by a magnetic force (Tsai et al. 2011).

Most early experimental and theoretical investigations of that problem were motivated by the fact that it may be considered as a canonical situation for understanding coalescence, especially the various stages of the drainage of the film located between the top part of the bubble and the fluid-fluid interface (Charles \& Mason 1960; Allan, Charles \& Mason 1961; Princen 1963; Princen \& Mason $1965 a, b)$. Hence they usually focused on creeping flow conditions and frequently considered the particular situation where the drop or bubble is made of the same fluid as one of the other two phases (see Chi \& Leal 1989 and Mohamed-Kassim \& Longmire 2004 for reviews). Then the problem began to be considered numerically, either in the limit of creeping motion using boundary integral techniques (Chi \& Leal 1989; Manga \& Stone 1995), or in presence of finite inertial effects by solving the full Navier-Stokes equations on a boundary-fitted grid (Shopov \& Minev 1992). Nevertheless only the early stages of the motion during which the drop approaches the fluid-fluid interface were considered in these computations because they could not deal with film break-up, nor with the subsequent topological changes of the flow. In the recent period, the development of high-speed video imaging techniques and particle image velocimetry has allowed the case of high-Reynolds-number gas bubbles crossing fluid-fluid interfaces to be investigated in more detail (Reiter \& Schwerdtfeger 1992a; Kemiha et al. 2007; Dietrich et al. 2008). In particular the evolution of the bubble shape and that of the column of heavy liquid it entrains under certain conditions were characterized in several regimes.

A closely related configuration that has received a great deal of attention is that of a rigid sphere approaching or crossing a horizontal fluid-fluid interface. Again this problem was initially considered in view of its connection with coalescence, and most investigations focused on the film-drainage configuration in which the sphere rests very close to an interface which only weakly deforms (Hartland 1968, 1969; Shah, Wasan \& Kintner 1972; Jones \& Wilson 1978; Smith \& Van de Ven 1984). Nevertheless some investigations rather considered, either experimentally (Maru, Wasan \& Kintner 1971) or numerically (Leal \& Lee 1982; Geller, Lee \& Leal 1986), situations in which the interface deformation may become large as time proceeds, the sphere then towing a long column or tail of heavy fluid with it. The case of heavy spheres settling across a sharp density interface separating two miscible fluids has also been considered experimentally, especially in connection with its relevance to the prediction of pollutant dispersion (Srdić-Mitrović, Mohamed \& Fernando 1999; Camassa et al. 2009). Finally it is worth mentioning that pseudo-three-phase systems with the same geometry have been used to study the entrainment of fluid or rigid particles by rising bubbles, especially in fluidized beds. In this case the two fluid layers are made of a single liquid and entrainment of the lower layer is quantified by marking it, for instance with dye or milk, and evaluating the volume of the displaced fluid. When the flow disturbance is close to that predicted by potential flow theory, this displaced 
volume may valuably be related to the classical concept of Darwin's drift (Eames \& Duursma 1997; Bush \& Eames 1998).

The present paper reports on a joint experimental and computational study of the above problem in the case of a single gas bubble crossing an interface between a lower phase made of water or water plus glycerin and an upper, slightly lighter, phase made of silicon oil. Varying the size of the bubble, the glycerin concentration and the characteristics of the silicon oil allows us to explore a broad range of physical conditions, leading to a variety of flow configurations and bubble shapes. The main purpose of this investigation is to obtain new insight into the key features of particular interest in this problem, namely the influence of film drainage on the bubble dynamics when it reaches the fluid-fluid interface, the final topology of the three phases, and, in cases where the bubble succeeds in crossing the interface, the evolution of its rise speed and of the volume of heavy fluid it entrains during its ascent in the upper fluid. Experimental data are obtained by means of high-speed video imaging and in some cases particle image velocimetry. Computations are based on the so-called volume of fluid approach in which the incompressible Navier-Stokes equations are solved on a fixed grid. As will be seen, the two approaches efficiently complement each other. For instance computations help reveal important details of the flow that cannot be obtained with the present optical technique, owing to limitations in its spatial resolution. They also give access to flow regions that may not be reached optically, due to the bubble shape (e.g. with toroidal or spherical caps bubbles with a concave base). Last but not least they guarantee that the various interfaces are surfactant-free and allow each physical property of the fluids to be varied independently for all others. However, three-phase flows involve complex small-scale phenomena such as film drainage, break-up and moving contact lines. Some of these phenomena may not be properly captured in computations, owing to the spatial cut-off introduced by the computational grid and to the approximate representation of interfacial forces. This is why direct computations of such flows cannot yet be performed in a blind manner and their results have to be compared with those of experiments to make sure that the former correctly predict the global dynamics revealed by the latter.

The paper is organized as follows. Sections 2 and 3 describe the experimental and computational approaches, respectively; specific technical details on the latter and validation tests are provided in three separate appendices. Section 4 presents an overview of the physical behaviours revealed by the entire set of experiments. In $\S 5$, we select six situations corresponding to contrasting flow conditions and bubble shapes and analyse each of them in detail with the help of experimental observations and computational predictions. Section 6 relies on some of the experimental and computational results and on a simple static model developed in appendix D to analyse the elementary mechanisms that drive the evolution of the system when the bubble reaches the fluid-fluid interface. Finally, $\$ 7$ summarizes the main findings obtained during this investigation and opens up some perspectives.

\section{Experimental device and measurement techniques}

Experiments are carried out at ambient temperature $\left(20 \pm 1{ }^{\circ} \mathrm{C}\right)$ in a glass tank $40 \mathrm{~cm}$ high with a $20 \mathrm{~cm} \times 20 \mathrm{~cm}$ square cross-section. Two sides of the tank are made of B270 Superwite ${ }^{\circledR}$ glass to limit optical distortions. The upper part of each vertical wall is treated with a Rain- $X^{\circledR}$ hydrophobic compound to avoid meniscus effects along the glass/water/oil contact line. The base of the tank is made of Plexiglas (PMMA); it is removable and comprises several interchangeable injection systems. After each series 

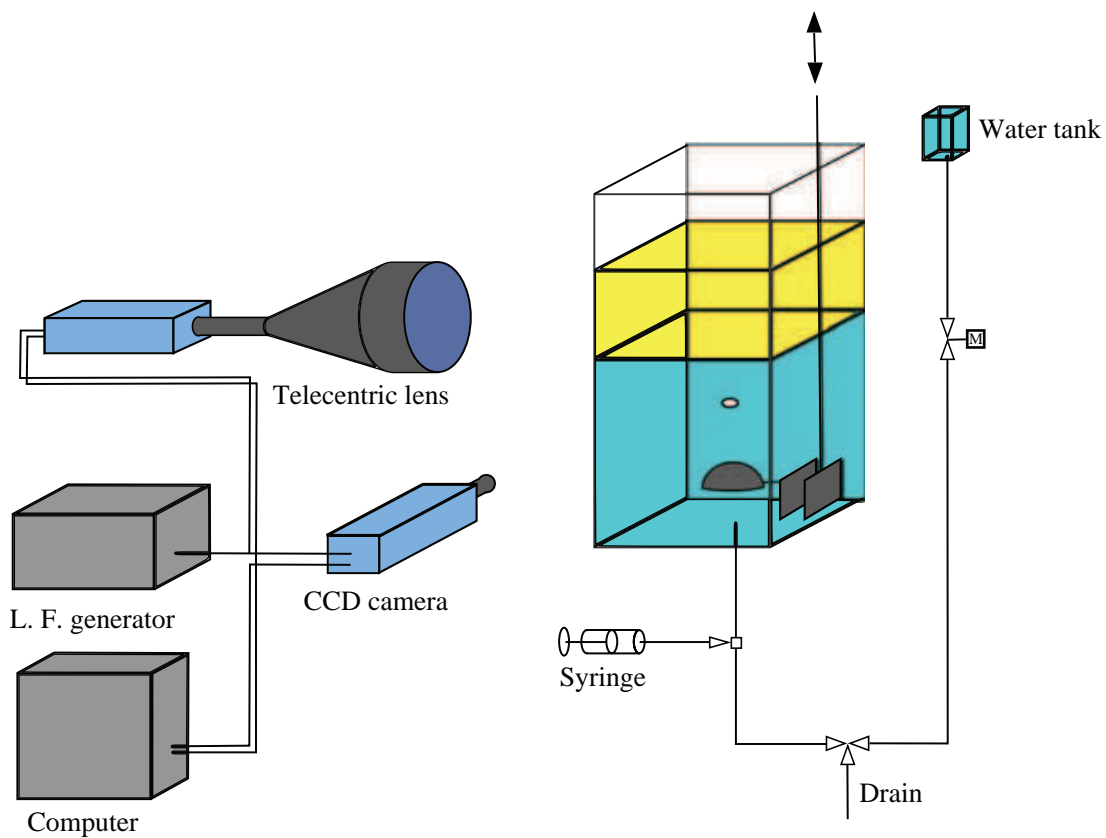

FIGURE 1. (Colour online) Sketch of the experimental device with the inverted beaker injection system. L.F., low frequency.

of measurements, each liquid is removed from the tank with a siphon at least every two days and stored in closed containers. The residual liquid layers adjacent to the liquid-liquid interface are thrown out. The tank is then washed with a detergent liquid, rinsed out with tap water a large number of times, and dried with a duster.

We basically employ two different gas injection techniques. One of them (shown schematically in figure 1) is inspired by the 'inverted beaker' used by Davies \& Taylor (1950) to produce large spherical cap bubbles. This device is made of a 'spoon' $60 \mathrm{~mm}$ in diameter, which may be rotated by hand about a horizontal axis. A controlled air volume is injected below the spoon through a syringe. Then the spoon is turned over to release the bubble. This device is suitable for generating spheroidal and spherical cap bubbles. In the second injection system, the air volume is initially entrapped in a closed cylinder and then rapidly injected manually in the tank by pushing a piston. This device is mostly used with large air volumes (typically $>1 \mathrm{~cm}^{3}$ ) to obtain toroidal bubbles. Based on the comparison between the injected volume of air and the optical determination (to be described below) of the bubble contour for small spherical bubbles, the uncertainty in the bubble equivalent diameter $d=(6 \mathscr{V} / \pi)^{1 / 3}$, where $\mathscr{V}$ is the bubble volume, was found to be $50 \mu \mathrm{m}$, so that the relative uncertainty in $d$ for bubbles injected with the first system ranges from $3 \%$ for the smallest of them $(d \approx 1.5 \mathrm{~mm})$ to less than $0.5 \%$ for the biggest $(d \approx 20 \mathrm{~mm})$.

Two high-speed digital Photon Lines PCO1200 HS cameras with a resolution of $1024 \times 1280$ pixels synchronized at a rate of 350 images per second are employed to visualize the bubble and the evolution of the various fluid-fluid interfaces. The two cameras are placed at right angles perpendicular to two of the vertical glass walls of the tank. We use backlighting to obtain the projections of the bubble and fluid-fluid interface shadow in the visualization planes (the schlieren technique). In 
order to reduce optical distortions, one of the cameras is equipped with an Opto Engineering telecentric lens TC 4M 120. The system is calibrated in such a way that each camera detects a fixed field of $108.9 \mathrm{~mm} \times 87.2 \mathrm{~mm}$. Contours of bubbles, droplets and columns of heavy liquid displaced through the upper fluid are detected on the images with a thresholding method followed by an erosion-dilatation process. Positions and surface elements identified on a given frame are then tracked in time using a maximum likelihood detection process. In some cases we also employ particle image velocimetry (PIV) to determine the velocity field past the bubble. For that purpose, a $2 \times 25 \mathrm{~mJ}$ Pegasus laser source lights up the two fluids seeded by Rhodamine-B molecules encapsulated in PMMA particles whose size is in the range $1-20 \mu \mathrm{m}$. The velocity fields are then extracted from intercorrelations of $(16 \times 16)$-pixel elements using the in-house software PIVIS.

In the experiments to be described below, the lower (and thus heavier) liquid is either tap water or a mixture of glycerin and tap water with two different volume fractions of glycerin, 85 or $95 \%$. No particular treatment is applied to water, so that surfactants are likely to be present and one may suspect them to somewhat lower the rise velocity of small spherical or spheroidal bubbles compared to theoretical predictions assuming a clean bubble surface; with usual surfactant concentrations, larger bubbles (say with $d>4 \mathrm{~mm}$ ) are known to be much less influenced by contamination. The upper (and thus lighter) liquid is silicon oil. Three different oils (47V10, 47V100 and 47V500 from Gaches Chimie company) are employed, with viscosities approximately ranging from 10 to 500 times that of water. The physical properties of all fluids were determined at room temperature $\left(20 \pm 1{ }^{\circ} \mathrm{C}\right)$. Viscosity and surface tension were obtained using a Bohlin cone-plate viscometer and a Wilhelmy plate device, respectively, while a drop shape analysis system, Kruss DSA100, based on the pendant-drop method was employed to measure the interfacial oil/water + glycerin tensions. The relative uncertainty in the viscosity is $5 \%$ for the least viscous oil and is a decreasing function of viscosity. The uncertainty in surface and interfacial tensions is $1.5 \mathrm{mN} \mathrm{m}^{-1}$, so that the corresponding relative uncertainty ranges from 2 (for the water/air system) to $11 \%$ (for the water/47V500 oil system). Density was determined by weighing a $100 \mathrm{ml}$ calibrated flask filled with the corresponding liquid on a precision balance with a $0.1 \mathrm{~g}$ accuracy, so that the corresponding relative uncertainty is $\sim 0.1 \%$. The various physical properties relevant to the systems described below are summarized in table 1 .

\section{Computational approach}

In the context of the one-fluid approach, a three-phase flow is considered as a mixture of three immiscible fluids. It may be characterized by the local volume fraction $C_{i}(i=1,2,3)$ of each of them and by density and viscosity fields that depend only on $C_{i}$ and on the corresponding intrinsic physical property of each fluid. Assuming all fluids to be Newtonian and the various interfaces to be sharp with uniform interfacial tensions, the system of equations governing the motion of an incompressible three-phase flow is then given by

$$
\begin{aligned}
\frac{\mathrm{D} C_{i}}{\mathrm{D} t} & =0 \quad \text { for } i=1,2,3, \\
\nabla \cdot \boldsymbol{U} & =0, \\
\varrho \frac{\mathrm{D} \boldsymbol{U}}{\mathrm{D} t} & =\varrho \boldsymbol{g}-\nabla P+\nabla \cdot\left\{\mu\left(\nabla \boldsymbol{U}+\nabla \boldsymbol{U}^{\mathrm{T}}\right)\right\}+\boldsymbol{T},
\end{aligned}
$$




$\begin{array}{lccc}\text { Liquid } & \begin{array}{c}\text { Density } \\ \left(\mathrm{kg} \mathrm{m}^{-3}\right)\end{array} & \begin{array}{c}\text { Viscosity } \\ (\mathrm{mPa} \mathrm{s})\end{array} & \begin{array}{c}\text { Surface tension } \\ \left(\mathrm{mN} \mathrm{m}^{-1}\right)\end{array} \\ \text { Water } & 997 & 1.002 & 69.4 \\ \text { 85\% glycerin + water } & 1211 & 102.6 & 48.7 \\ \text { 95\% glycerin + water } & 1244 & 550.1 & 45.2 \\ \text { 47V10 silicon oil } & 932 & 9.6 & 20.2 \\ \text { 47V100 silicon oil } & 961 & 113.8 & 20.7 \\ \text { 47V500 silicon oil } & 965 & 530.7 & 21.0 \\ \text { Interfacial tension }\left(\mathrm{mN} \mathrm{m}^{-1}\right) & 47 \mathrm{~V} 10 \text { oil } & 47 \mathrm{~V} 100 \text { oil } & 47 \mathrm{~V} 500 \text { oil } \\ \text { Water } & 19.7 & 14.3 & 13.7 \\ \text { 85\% glycerin + water } & 28.8 & 30.0 & 29.9 \\ 95 \% \text { glycerin + water } & 27.8 & 27.1 & 28.0\end{array}$

TABLE 1. Physical properties of the various liquids measured at $20 \pm 1{ }^{\circ} \mathrm{C}$.

where $\mathrm{D} / \mathrm{D} t=\partial / \partial t+\boldsymbol{U} \cdot \boldsymbol{\nabla}$ denotes the material derivative, $\boldsymbol{T}$ is the capillary force density, and the density and viscosity of the mixture are related to the volume fraction of each phase through the linear laws

$$
\varrho=\sum_{i=1}^{3} C_{i} \varrho_{i}, \quad \mu=\sum_{i=1}^{3} C_{i} \mu_{i} .
$$

Note that the first of (3.4) is an exact result while the second is just an ad hoc interpolation formula. The JADIM code developed at IMFT solves the above set of equations with a capillary force density defined as

$$
\boldsymbol{T}=-\frac{1}{2} \sum_{i=1}^{3} \sum_{j=1}^{3}\left(C_{i}+C_{j}\right)^{2} \sigma_{i j} \nabla \cdot\left[\frac{\nabla C_{i j}}{\left\|\nabla C_{i j}\right\|}\right] \nabla C_{i j},
$$

where $C_{i j}=C_{i} /\left(C_{i}+C_{j}\right)=1-C_{j i}$. The derivation of (3.5), which is an extension of the continuum surface force (CSF) formulation (Brackbill, Kothe \& Zemach 1992), is detailed in appendix A; comparison with alternative formulations is also discussed there.

The code is based on a finite-volume discretization combined with a Runge-Kutta/Crank-Nicolson time-advancement scheme; incompressibility is enforced via a projection method. Centred schemes are used to approach the various spatial derivatives involved in (3.3) while the solution of (3.1) is based on a direction-splitted version of Zalesak's Flux-Corrected Transport algorithm (Zalesak 1979). The full numerical approach has been extensively described by Bonometti \& Magnaudet (2007) (see also Bonometti \& Magnaudet 2006) and will not be repeated here. However, the way we deal with the issue of volume conservation of each phase in a three-phase system deserves some specific comments, which are provided in appendix B.

Throughout the paper, axisymmetric computations are performed within a vertical cylindrical domain with a $6 d$ radius and a $12 d$ height, the initial fluid-fluid interface standing $7 d$ from the top of the domain. The grid employs 1200 cells uniformly distributed in the vertical direction. In the horizontal direction, 200 grid points are uniformly distributed in the central region extending up to $2 d$ from the axis, while another 100 points are distributed in the outer region following an arithmetic law. The present resolution, with a hundred cells per bubble diameter, was used by Bonometti \& 
Magnaudet $(2006,2007)$ who found it to provide grid-independent results for various types of bubbles rising in a Newtonian fluid, including toroidal bubbles; this is why it is selected again here. Free-slip boundary conditions are imposed on the top, bottom and lateral boundaries, so that the fluid entrained upwards by the bubble slowly goes down near the lateral boundary. Computations are stopped before the bubble gets close to the upper boundary, to avoid contamination of results by confinement effects.

The code was extensively validated in the past in various two-phase configurations. Simple three-phase configurations for which a theoretical solution exists, such as the spreading of a small lens at the interface between two liquids (de Gennes, BrochardWyart \& Quéré 2004), were also considered. These tests showed that the theoretical shape of the lens is recovered under various conditions of spreading, which validates the above formulation for the capillary force $\boldsymbol{T}$. An additional test case in a physical situation close to those under focus here and employing the grid characteristics described above is detailed in appendix $C$. This test deals with the early evolution of low-Reynolds-number buoyancy-driven drops of various viscosities rising toward a horizontal interface separating two fluids with different viscosities and densities, a configuration that was computed by Manga \& Stone (1995) using a boundary integral method (BIM). The results of both approaches turn out to be in excellent agreement until the drop gets very close to the interface between the lower and upper fluids. More precisely, with low-viscosity drops behaving very similarly to bubbles, differences from the predictions of Manga and Stone start to be significant when the thickness of the film separating the top of the drop from the upper fluid becomes $\lesssim 0.04 d$. These differences result from two phenomena, both of which are related to the finite thickness of the interfaces in the present approach. First, the capillary force density (3.5) involves the gradients of $C_{i}$ and then spreads out over several cells across an interface. Second, the interpolation law (3.4) used to determine the local viscosity implies that, within an interfacial region, viscosity takes values intermediate between those of the two fluids in contact. Both phenomena become increasingly important within the film as it thins: being bounded by two interfaces, the flow in the gap experiences some artificial capillary force and the viscosity is no longer that of the actual fluid. The consequences of these artifacts will be seen in more detail in $\S 5$.

\section{Overview of experimental observations}

We start by providing a qualitative survey of the evolution of the three-phase system as its characteristic parameters are varied. For this purpose, a prerequisite is the definition of a proper set of dimensionless characteristic numbers. General three-phase systems involving Newtonian fluids are characterized by nine physical properties, namely three densities $\left(\rho_{i}, i=1,2,3\right)$, three viscosities $\left(\mu_{i}\right)$ and three interfacial tensions $\left(\sigma_{i j}\right.$ with $j \neq i$ and $\sigma_{i j}=\sigma_{i j}$ ). Moreover the dynamical problem depends on gravity $g$ and assuming that the bubble is initially spherical and located far from the liquid-liquid interface within a flow domain extending up to infinity, on a single length scale, for instance the bubble equivalent diameter $d$. As these eleven quantities involve three fundamental units (mass, time and length), the problem may be characterized with eight independent dimensionless parameters. However, since the bubble viscosity and density are negligibly small compared to those of the various liquids we use, they may be removed from the list of relevant quantities, which reduces the number of independent dimensionless parameters to six. Let fluid 1 (respectively 3) refer to the lower (respectively upper) liquid as in the previous section. Then, defining all parameters with respect to the properties of 
fluid 1, we may select $R=\left(\rho_{1}-\rho_{3}\right) / \rho_{1}, \Lambda=\mu_{3} / \mu_{1}, I=\sigma_{13} / \sigma_{12}, S=\sigma_{23} / \sigma_{12}, B o=$ $\rho_{1} g d^{2} / \sigma_{12}, A r=\rho_{1}\left(g d^{3}\right)^{1 / 2} / \mu_{1}$. The Bond (or Eötvös) number Bo compares buoyancy effects to capillary effects while the Archimedes number $A r$ may be thought of as a Reynolds number based on the gravitational velocity $(g d)^{1 / 2}$. The problem is then entirely defined by the set of parameters $(R, \Lambda, I, S, B o, A r)$.

Given the values of the physical properties reported in table 1 , the viscosity ratio $\Lambda$ varies by more than four orders of magnitude through the whole set of experiments, from 0.0175 (when the lower liquid is a $95 \%$ glycerin + water mixture and the upper liquid is the 47V10 silicon oil) to 530 with the water/47V500 silicon oil system. The density ratio $R$ varies from 0.036 to 0.251 (note that, owing to its definition, $R$ always satisfies the constraint $0 \leqslant R \leqslant 1$ ). The parameter $I$, a dimensionless measure of the strength of interfacial effects at the fluid-fluid interface, varies by a factor of three between series $\mathrm{A}$ or $\mathrm{B}$ and series $\mathrm{D}(0.21 \leqslant I \leqslant 0.62)$, while the ratio $S$ of surface tensions in the upper and lower fluids experiences a more modest variation $(0.29 \leqslant S \leqslant 0.465)$. In view of future discussions, it is also useful to introduce the Archimedes and Bond numbers in the upper fluid, $A r_{u}$ and $B o_{u}$, defined as $A r_{u}=(1-R) A r / \Lambda$ and $B o_{u}=(1-R) B o / S$, respectively.

Images of some of the bubbles emerging in the upper fluid and of the associated entrained volume of heavy fluid (if any) are displayed in figure 2. Each twodimensional diagram in that figure corresponds to a given set of fluids (i.e. given values of $R, \Lambda, I$ and $S$ ); the Bond and Archimedes numbers in each series are varied by generating bubbles of increasing volume, i.e. increasing $d$. As suggested by figure 2, small enough bubbles, typically those with $B o<5$ in series A or $B o<7$ in series $\mathrm{B}$, remain trapped at the interface between the two liquids during the entire period of observation (typically $1 \mathrm{mn}$ in this range of $B o$ ). That is, no macroscopic change has occurred in the system at the time we stop recording images but this obviously does not rule out the possibility of later changes, since these bubbles are expected to be covered by a very thin film of heavy liquid that should eventually be drained, leading to the release of the bubble after the film has ruptured. This entrapment corresponds to situations in which capillary effects result in a downward force capable of balancing the upward buoyancy force. Although the full film-drainage problem has been worked out in great detail in the low-Reynolds-number limit (Princen 1963; Princen \& Mason 1965a,b), it does not have a general tractable theoretical solution, even in that limit. A crude criterion predicting conditions under which bubble entrapment occurs was derived by Greene, Chen \& Conlin (1988). Based on the idea that a bubble cannot be stopped at the interface if the buoyancy force it experiences in the light fluid exceeds the maximum possible interfacial capillary force (reached when the contact line lies in the horizontal mid-plane of the bubble and the meniscus is vertical), this criterion is in the present notation given by Bo $<6 I /(1-R)$ if the bubble is assumed to be spherical. It suggests that bubbles of series A and B should not be stopped near the undisturbed position of the interface for $B o>5$ approximately, while those in series C (respectively D) should not be stopped for $B o>1.8$ (respectively 1.3). According to figure 2, this criterion predicts the trapped/untrapped transition reasonably well. We shall consider the mechanisms underlying this transition in more detail in $\S 6$.

Bubbles that are not trapped near the interface then rise in the upper liquid and tow a column (or tail) of heavy liquid for some time. Experimental observations suggest that after the bubble has travelled a distance of a few $d$ above the initial position of the interface, this column is most often directly attached to the rear part of the bubble, i.e. no film is discerned on the front part of the bubble at this late stage, 

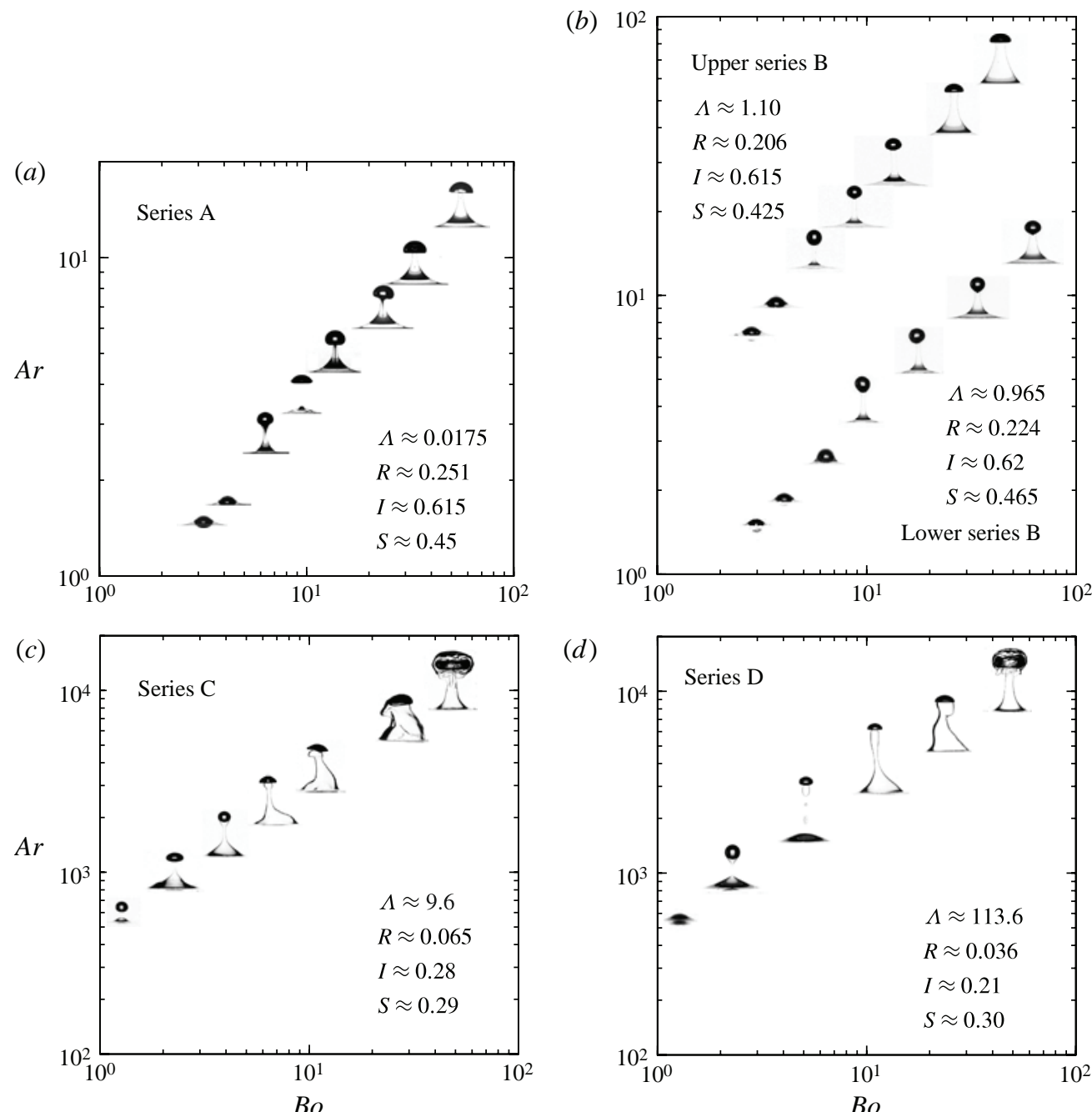

FIGURE 2. Some selected shapes of bubbles and entrained columns of heavy fluid in five series of experiments (only the bubble size is varied in each series). (a) Series A (95\% glycerin + water/47V10 oil, $3 \leqslant B o \leqslant 50,1.5 \leqslant A r \leqslant 12$, i.e. $\left.5 \leqslant B o_{u} \leqslant 83,64 \leqslant A r_{u} \leqslant 514\right)$. (b) Lower series B (95\% glycerin + water/47V500 oil, $3.8 \leqslant B o \leqslant 55,1.3 \leqslant A r \leqslant 12$, i.e. $\left.6.3 \leqslant B o_{u} \leqslant 92,1.0 \leqslant A r_{u} \leqslant 9.6\right) ;$ upper series $\mathrm{B}(85 \%$ glycerin + water/47V100 oil, $2.1 \leqslant$ $B o \leqslant 50,7.0 \leqslant A r \leqslant 60$, i.e. $3.9 \leqslant B o_{u} \leqslant 93,5.0 \leqslant A r_{u} \leqslant 43$ ). (c) Series C (water/47V10 oil, $1.2 \leqslant B o \leqslant 50,550 \leqslant A r \leqslant 8.0 \times 10^{3}$, i.e. $\left.3.9 \leqslant B o_{u} \leqslant 160,54 \leqslant A r_{u} \leqslant 780\right)$. (d) Series D (water/47V100 oil, $1.3 \leqslant B o \leqslant 52,500 \leqslant A r \leqslant 7.4 \times 10^{3}$, i.e. $4.2 \leqslant B o_{u} \leqslant 167,1.6 \times 10^{3} \leqslant$ $\left.A r_{u} \leqslant 23.8 \times 10^{3}\right)$. In each image, the vertical axis is positioned on the corresponding value of $B o$ while the horizontal trace of the flat fluid-fluid interface is positioned on the corresponding value of $A r$.

implying that film drainage has already been completed and that a moving contact line subsequently develops between the bubble and the two liquids. A noticeable exception is that of toroidal bubbles, which turn out to remain completely encapsulated in a thick film of heavy liquid. The column may sometimes break up into droplets when $\Lambda \gg 1$ (e.g. $B o \approx 5$ and $A r \approx 1.3 \times 10^{3}$ in series D) because it is then strongly sheared by the 
upper fluid that hardly deforms. In the opposite case where the upper liquid deforms much more easily than the lower one $(\Lambda \ll 1)$, bubbles are accelerated after they have crossed the interface (although the buoyancy force is somewhat less than in the lower fluid). This acceleration may totally suppress the entrained column because it may break the bubble when it is close to emerging entirely from the interface, forcing its former rear part to remain trapped between the two liquids (e.g. $B o \approx 9$ and $A r \approx 3.2$ in series A).

Depending on their final Bond and Reynolds numbers, rising bubbles may look like oblate spheroids (left part of series C and D or central part of series B), exhibit a marked tail (central part of series A or right part of the lower series B), look like spherical caps (right part of series A and of the upper series B, central part of series $\mathrm{C}$ and $\mathrm{D}$ ) or, for large enough Bond and Archimedes numbers, adopt a toroidal shape (upper right corner of series $\mathrm{C}$ and $\mathrm{D}$ ). According to the position at which bubbles approximately switch from one shape to the other, the transition from spheroidal to spherical cap bubbles may be estimated to take place at $B o_{u} \approx 20$ provided $A r_{u} \geqslant 20$, while that from spherical cap to toroidal bubbles takes place at $B o_{u} \approx 125$ and $A r_{u} \approx 50$. As it is directly related to the width of the wake, the head of the entrained column is significantly thicker with spherical caps than with spheroidal bubbles, resulting in a larger displaced volume. Also, for a given Bond number and position above the undisturbed interface, the larger the Archimedes number (and thus the Reynolds number) in the upper fluid, the thinner the wake and hence the smaller the displaced volume (compare the two snapshots in the upper right corner of series $\mathrm{A}$ and $\mathrm{B}$, where $A r_{u}$ is $\sim 12$ times larger in the former series). Most configurations displayed in figure 2 are axisymmetric. Fully three-dimensional configurations are only observed in series $\mathrm{C}$ and $\mathrm{D}$ when $A r>10^{3}$. In this regime, which also corresponds to $O\left(10^{3}\right)$ Reynolds numbers, the wake of axisymmetric bluff bodies is known to be three-dimensional, whether they are spheres or disks obeying a no-slip condition (Natarajan \& Acrivos 1993) or stress-free spheroidal bubbles with a sufficient oblateness (Magnaudet \& Mougin 2007). When the corresponding bubbles rise in the upper fluid, their actual Reynolds number drops by one (respectively two) order of magnitude in series C (respectively D) but in most cases this is still sufficient for the wake to remain unstable, resulting in tortuous bubble shapes and columns of entrained fluid. Remarkably, for a given Bond number, the bubble and displaced volume geometries in series $\mathrm{C}$ and D are quite similar as soon as Bo exceeds some units, although the actual Archimedes number in the upper liquid differs by one order of magnitude between the two series. This is an indication that the flow about the bubble is only weakly affected by viscous effects in these strongly inertial regimes.

\section{Analysis of experimental and computational results in some selected situations}

To explore the dynamics of the three-phase system in more detail, we select some situations within figure 2. One of them is typical of small bubbles (with $B o=O(1))$ that remain trapped at the interface throughout the period of observation. The following two are characterized by moderate, say $O(10)$, Bond numbers and result in bubbles with a more or less spheroidal shape, possibly with some cusp at the rear. They differ in the strength of inertial effects, which are weak in one case while they dominate in the other. Two other situations belong to regimes characterized by Bond numbers of several tens where the bubble eventually takes a spherical cap shape; the magnitude of inertial effects in the upper fluid differs by one order of magnitude 
(a)

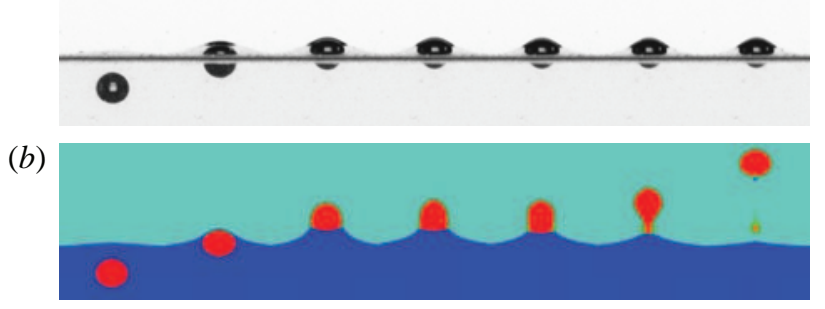

FIGURE 3. (Colour online) Evolution of the three-phase system for a bubble corresponding to $B o=3.0$ and $A r=7.65$ in the upper series B. Comparison between $(a)$ the experimental sequence and $(b)$ computational predictions. The dimensionless time period $(g / d)^{1 / 2} \Delta t$ between two consecutive images is $\sim 4.1$.

between these two cases, yielding significant differences in their evolutions. Finally, the last situation we consider is typical of the large toroidal bubbles that develop when the Bond and Archimedes numbers are large enough.

Experimentally, the rise speed is obtained by tracking the successive positions of the bubble's uppermost point with the image processing technique described in $\S 2$. Unless stated otherwise, we prefer to define the velocity $V$ at this point rather than that of the bubble centroid because some parts of the surface may not be visible in experimental sequences involving dimpled bubbles, leading to errors in the determination of the centroid. In computations, spherical bubbles are released from rest with their centre located a distance $3 d$ below the undisturbed fluid-fluid interface; this is why in the evolutions of the rise speed displayed below, this quantity is first seen to increase briefly, before reaching a broad maximum (see e.g. figures 5 and 7). Results are presented in dimensionless form using the bubble's initial diameter $d$ and the bubble volume $\mathscr{V}=\pi d^{3} / 6$ to normalize positions and volumes, respectively, while velocities and times are normalized by the gravitational scales $(g d)^{1 / 2}$ and $(d / g)^{1 / 2}$, respectively.

\subsection{A small bubble trapped at the interface}

We start by considering a small bubble with $B o=3.0$ and $A r=7.65$ in the upper series B $(\Lambda=1.11)$. As revealed by figure $3(a)$, this corresponds to a case where, once the bubble has reached the region of the fluid-fluid interface, it remains trapped there throughout the rest of the period of observation because the film of heavy liquid that covers it has not yet been completely drained at the end of the sequence. Although the bubble is almost spherical before it reaches the interface region, its 'final' shape exhibits a marked top-bottom asymmetry with a fairly flat top region and a more rounded bottom part. Since the whole system is almost at rest, this is an indication that the hydrostatic pressure variations influence the local curvature of the bubble surface. Indeed, comparing the capillary length $l_{c}=\left(\sigma_{12} / \rho_{1} g\right)^{1 / 2}$ with the bubble radius reveals that the ratio of both quantities is $d /\left(2 l_{c}\right)=B o^{1 / 2} / 2 \approx 0.87$.

According to figure 3 , computational predictions differ dramatically from observations except during the early stages of the motion. The departure becomes visible in the third snapshot, when the computed bubble is no longer completely immersed in the lower fluid. Indeed the film that was still covering its top part in the previous snapshot has ruptured in the meantime. This is no surprise since the film was already very thin at that time (significantly less than $d / 10$ ) and the local grid spacing is only $d / 100$, implying that only a few grid cells lay in the gap. Then, for reasons 
(a)

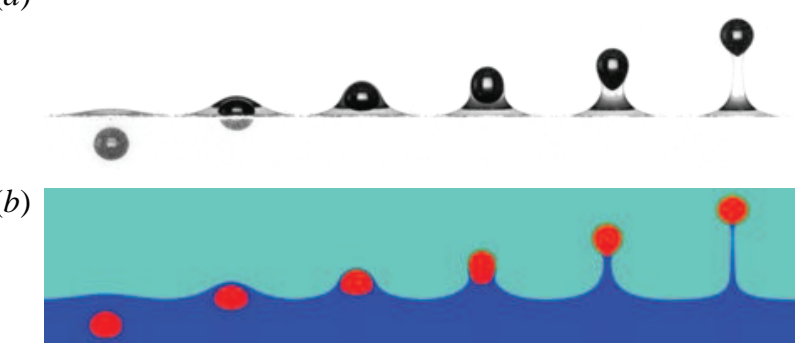

FIGURE 4. (Colour online) Evolution of the three-phase system for a bubble corresponding to $B o=13.2$ and $A r=4.1$ in the lower series B. Comparison between $(a)$ the experimental sequence and $(b)$ computational predictions. The dimensionless time period separating two consecutive images is $\sim 4.3$.

similar to those discussed in $\S 3$ (see also appendix C), the flow in the gap is poorly described and numerical break-up soon occurs.

Although this computed evolution disagrees with the experimental observations, it deserves a few comments. As shown in figure 3, the computed bubble next spends a long time 'floating' on the interface (snapshots 3-6). A large meniscus first develops. Then it slowly recedes and a neck forms at the bottom of the bubble whose shape tends gradually toward that of a 'hot air balloon'. This particular shape may be shown to be the only one that, given the values of $I, S, R$ and $B o$, satisfies both the Neumann condition expressing the equilibrium of the contact line (de Gennes et al. 2004) and the overall momentum balance expressing the vertical equilibrium of the bubble. In other words, if the bubble were released from rest right at the interface, it would take a shape and generate a meniscus very similar to those displayed in the last-but-one snapshot of the computational sequence in figure $3(b)$ and would stay there forever. However, in the present computation, the bubble is released well below the interface and the decrease in the potential energy of the whole system as it rises makes the kinetic energy of the fluid non-zero at the time it starts floating, even though some of this potential energy is dissipated by viscous effects and part of it is converted into interfacial energy through the increase of the bubble and fluid-fluid interface areas. This is why the floating configuration is only a transient in the present case and pinch-off finally takes place at the bottom of the bubble, entailing its release in the upper fluid.

\subsection{Spheroidal bubbles}

We now consider a situation belonging to the lower series $\mathrm{B}$ with a bubble nearly twice as big as that of the previous section. Hence the Bond number is $B o=13.2$ but the Archimedes number is only $A r=4.1$ owing to the high viscosity of the lower liquid. According to the experimental sequence displayed in figure 4(a), the bubble now succeeds in crossing the initial position of the fluid-fluid interface, although it is still covered by a film for some time. The film is seen to break up between the third and fourth snapshots, after which the bubble starts to become more elongated and to tow a column of heavy fluid. As the top of the column recedes along the bubble surface, the rear of the bubble becomes more pointed or even exhibits a small tip, owing to the stretching resulting from the combined effect of the bubble ascent and the recession of the column (snapshots 5-6). The column eventually separates from the bubble which then rises freely in the upper fluid. 

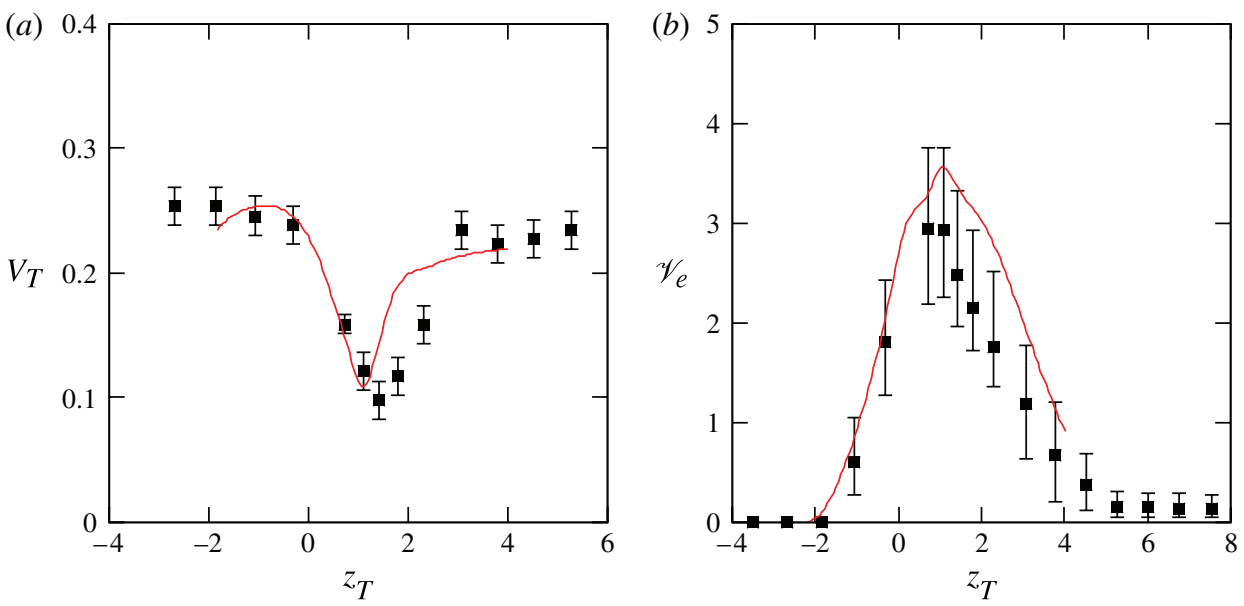

FIGURE 5. (Colour online) Evolution of $(a)$ the normalized rise speed $V_{T}$ and $(b)$ the displaced volume $\mathscr{V}_{e}$ as a function of the dimensionless bubble position $z_{T}$ for the situation considered in figure 4: $\mathbf{\square}$, experiment; solid line, computations. The error bars on $V_{T}$ result from the \pm 1 pixel uncertainty in the displacement of the uppermost point of the bubble between two successive images, while those on $\mathscr{V}_{e}$ result from the 3-pixel uncertainty in the local radius of the entrained column.

The computed evolution correctly reproduces the various stages of the experimental sequence. In the late stages, the entrained column is noticeably thinner than its experimental counterpart at the same instant in time, but this is essentially because the computed bubble has travelled a somewhat larger distance (see below). Figure 5(a) shows how the normalized rise speed $V_{T}=V /(g d)^{1 / 2}$ evolves as a function of the dimensionless position $z_{T}$ of the top of the bubble above the undisturbed position of the interface. Starting from its steady value in the lower fluid, $V_{T}$ slightly reduces when the top of the bubble approaches the fluid-fluid interface. Then it drops to less than half its initial value at the end of the period when the bubble is still covered by the film. After the film has ruptured, $V_{T}$ increases monotonically over a period of time during which the bubble crosses a distance about twice its diameter, after which it almost reaches its new terminal value. The latter is somewhat less than it was in the lower fluid, essentially because of the reduction of the buoyancy force $(R=0.224)$. According to figure 5(a), the bubble Reynolds number $\operatorname{Re}=A r V_{T}$ and Weber number $W e=B o V_{T}^{2}$ are of $O(1)$ in both fluids. A theoretical prediction for the terminal velocity taking into account inertial corrections corresponding to small $R e$ and We was derived by Taylor \& Acrivos (1964) using matched asymptotic expansions. It is of interest to examine how it compares with the experimental values of $V_{T}$. Equating the buoyancy force with the drag force predicted by Taylor \& Acrivos in the case of a massless drop with zero internal viscosity yields the nonlinear equation

$$
V_{T}\left\{1+\frac{R e}{8}+\frac{R e^{2}}{40} \ln \frac{R e}{2}+\frac{W e}{12}\right\}=\frac{A r}{12} .
$$

With present values of $A r$ and $B o,(5.1)$ predicts $V_{T} \approx 0.278$, which is in fairly good agreement with, albeit slightly larger than, the initial value of $V_{T}\left(V_{T} \approx 0.26\right)$ reported in figure $5(a)$. The slight difference may be attributed to the contamination effects that tend to lower the rise speed. Note that if inertial corrections were neglected in 
(5.1), one would predict $V_{T} \approx 0.342$, which clearly overestimates the actual rise speed. Replacing $A r$ by $A r_{u}$ and $B o$ by $B o_{u}$ in (5.1), the bubble terminal velocity in the upper fluid is found to be $V_{T} \approx 0.230$ which is in excellent agreement with the experimental and computational values (contamination effects may decrease as the bubble rises in the upper fluid since silicon oils are known to be non-polar). The drop experienced by the rise speed when the bubble enters the region of the fluid-fluid interface is correctly predicted in the computation. However, for reasons already discussed above, the film that covers the top of the bubble breaks somewhat too early, preventing $V_{T}$ from decreasing as much as it should and forcing it to start re-increasing slightly too early. The shift of the acceleration period toward lower positions could probably be resolved by a local increase of the grid resolution that would allow the film to subsist longer. However, we did not explore this possibility as we wish to determine how the computational approach deals with all physical situations under consideration with a single prescribed spatial resolution.

The volume of heavy fluid dragged into the upper fluid is a quantity of primary interest in three-phase systems. Depending on the context, it may for instance determine the mixing efficiency of the process or the amount of fluid that risks being projected if there is a free surface on top of the whole system (e.g. in iron processing where the upper layer is made of slag as discussed by Poggi et al. 1969, Reiter \& Schwerdtfeger 1992a,b, Kobayashi 1993). We define this displaced volume as that of heavy fluid located above the position of the initial horizontal interface (i.e. we do not take into account the tiny reflux induced by mass conservation at large distance from the bubble path). Hereinafter this displaced volume is normalized by the bubble volume $\mathscr{V}$, defining the dimensionless displaced volume $\mathscr{V}_{e}$. As figure $5(b)$ shows, $\mathscr{V}_{e}$ reaches a maximum $\sim 3$ for $z_{T} \approx 1$, i.e. when the top of the bubble is about one diameter above the undisturbed interface (third snapshot in figure 4). Then $\mathscr{V}_{e}$ decreases continuously until it reaches a constant small-but-non-zero value $(\sim 0.15)$ for $z_{T} \geqslant 5$. This non-zero final value indicates that a small volume of lower liquid remains permanently entrained by the bubble. Note that, counterintuitively, the comparison of figures 4 and $5(b)$ at various bubble positions indicates that the higher the liquid column entrained by the bubble, the smaller the total entrained volume. This is because the major contribution to the entrained volume comes from the region close to the fluid-fluid interface (where the surface of the displaced volume has a large radius) and not from the most visible part of the column (that attached to the rear of the bubble), whose radius is small.

We turn to a configuration belonging to series $\mathrm{A}$ with almost the same values of the Bond and Archimedes numbers $(B o=13.3$ and $A r=4.15)$ as the one we just described; the main difference lies in the much lower viscosity of the upper fluid which, as will be seen, induces a markedly different evolution. As may be seen in figure $6(a)$, the first part of the sequence is similar to that observed in the previous case: after the bubble has started to deform the fluid-fluid interface, the film on its top part is quickly drained and the bubble starts to emerge in the upper fluid with a prolate shape. Then, as the vaguely hemispherical head of the bubble rises, a thin and long tail of air develops behind it, surrounded by a column of heavy fluid. This is because the low viscosity of the upper fluid allows the head of the bubble to rise fast while the part that is still in contact with the lower fluid is forced to rise much more slowly. After some time, the column of heavy fluid breaks right at the rear of the bubble head and starts receding. This in turn breaks the top of the bubble tail, part of which escapes from the entrained column and starts rising as an autonomous secondary bubble. The rest of the bubble tail recedes with the entrained column and eventually 
(a)

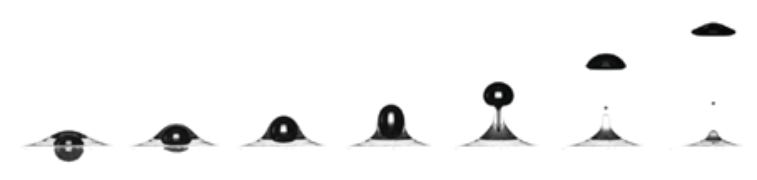

(b)

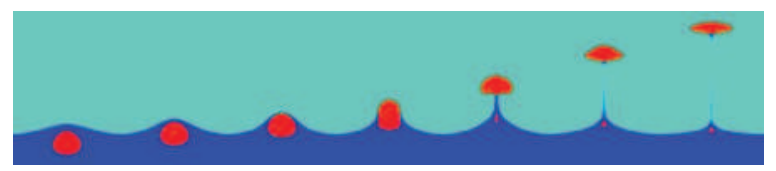

FIGURE 6. (Colour online) Evolution of the three-phase system for a bubble corresponding to $B o=13.3$ and $A r=4.15$ in series A. Comparison between $(a)$ the experimental sequence and $(b)$ computational predictions. The dimensionless time period separating two consecutive images is $\sim 1.6$.
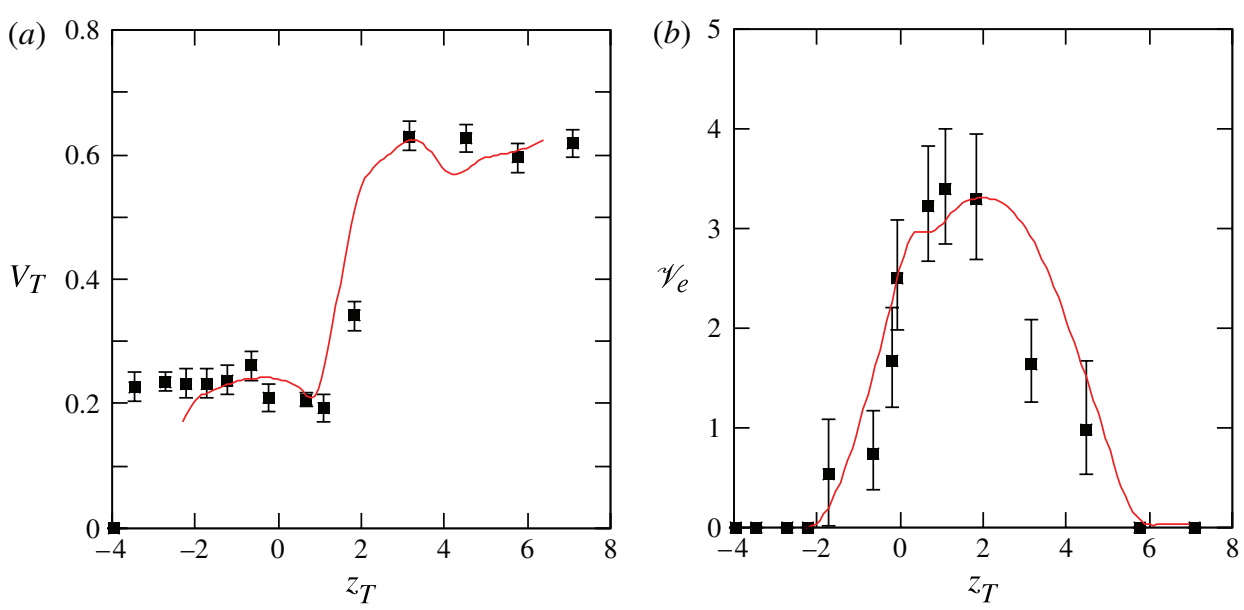

FIGURE 7. (Colour online) Evolution of ( $a$ ) the normalized rise speed $V_{T}$ and $(b)$ the displaced volume $\mathscr{V}_{e}$ for the bubble considered in figure 6 . See figure 5 for legend.

remains stuck just below the fluid-fluid interface. Note that after having released the column of heavy fluid, the main bubble undergoes significant shape oscillations.

The corresponding computational predictions are displayed in figure $6(b)$. The predicted shape and position of the main bubble are in good agreement with the experiments at each step of the sequence, although some differences may be noticed after the entrained column starts to form. In particular, the computations predict that a very thin column remains towed by the bubble at the end of the sequence although no such trend is detected experimentally. We also notice that the small rising bubble resulting from the break-up of the tail is not captured. Again, most of these secondary discrepancies could probably be removed by using a finer grid, but we did not explore this option.

Figure $7(a)$ shows how the normalized rise speed $V_{T}$ of the bubble evolves. Here again, after starting from an initial steady-state value accurately predicted by (5.1), $V_{T}$ experiences some transient drop when the bubble starts to cross the fluid-fluid interface, owing to the retarding effect of the film. Then $V_{T}$ increases by a factor of three while the bubble rises a distance less than $2 d$. This strong acceleration, after 
which $V_{T}$ stabilizes itself at a value $\sim 0.6$, is of course due to the large viscosity contrast between the two liquids $(\Lambda=0.0175)$. However, the evolution of the bubble shape, which becomes very oblate (the bubble aspect ratio is $\sim 3.4$ in the last snapshot of figure 6) and hence forces it to displace a large quantity of fluid as it rises, limits the increase of $V_{T}$ well below the value it would reach if the bubble had kept its initial spherical shape (in which case the final rise speed would typically be $1 / \Lambda$ larger than that in the lower fluid). According to the terminal value of $V_{T}$ at the end of the sequence, the final bubble Weber number $W e=V_{T}^{2} B o_{u}$ is $\sim 8.8$ and the final Reynolds number $\operatorname{Re}=V_{T} A r_{u}$ is $\sim 110$. Although the latter value might suggest that the high-Re theory of Moore (1965) could be used to predict the terminal rise speed, this is actually not the case. The reason is that the bubble shape must be an oblate spheroid for this theory to hold, and such a shape exists in the high-Reynolds-number limit only if the Weber number is less than a critical value close to 3.23 (Miksis, Vanden-Broeck \& Keller 1981; Meiron 1989). The present bubble is well beyond this limit, which explains why it oscillates while rising, and no theory is available to predict the rise speed under such conditions. According to figure $7(a)$, the computation correctly predicts the evolution of $V_{T}$, although there is still some shift on the position at which its sharp increase occurs. This shift is of course reminiscent of that observed in figure $5(a)$ and certainly has the same origin. During the final stage of the sequence, the predicted rise speed is seen to exhibit oscillations which are of course coupled to those of the bubble shape. The average value of $V_{T}$ in this final stage agrees well with that deduced from experiments.

Figure $7(b)$ displays the evolution of the displaced volume. This volume first increases until it reaches a maximum about three times that of the bubble when $z_{T} \approx 2$. Then the entrained column starts receding, forcing $\mathscr{V}_{e}$ to decrease and eventually return to zero for $z_{T} \approx 6$. Note that the net buoyancy force acting on the bubble + column system is positive only if $\mathscr{V}_{e}<(1-R) / R \approx 3$. Hence, to sustain the bubble rise, the normalized entrained volume cannot exceed $(1-R) / R$, except during some transient stage. Figure $7(b)$ also shows that the computed evolution of the displaced volume is in close agreement with the experimental determination, although the retraction seems somewhat too slow, a direct consequence of the thin column that remains attached to the bubble in the late stages of the sequence.

\subsection{Spherical cap bubbles}

Let us now consider another bubble in series A with a diameter twice that of the previous one, i.e. $B o=52.9$ and $A r=11.7$. The corresponding sequence is displayed in figure 8. Here, since surface tension effects are weak, the rear part of the bubble is markedly dimpled when it reaches the fluid-fluid interface. After the short and thick tail it exhibits for some time has retracted, the bubble takes a final spherical cap shape with an angle close to $120^{\circ}$. Given this shape, the column of liquid it entrains is much thicker than in the previous case, although it gets thinner as the travelled distance increases and eventually breaks. The whole evolution is correctly predicted by the computations, including the transient presence of the aforementioned short thick tail (keep in mind that what is revealed by the experimental photographs is a side view of the bubble surface, while the computational snapshots show the bubble cross-section). The neck exhibited by the entrained column in the last experimental snapshot is also correctly captured.

Figure $9(a)$ shows how the normalized rise velocity $V_{T}$ of the top of the bubble evolves. Remarkably, $V_{T}$ does not experience any jump (only a small bump) after the bubble has crossed the interface, although all fluid properties are similar to those of 
(a)

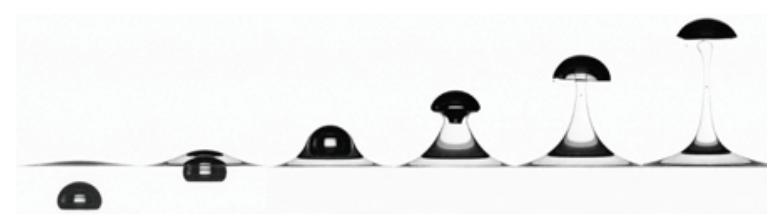

(b)

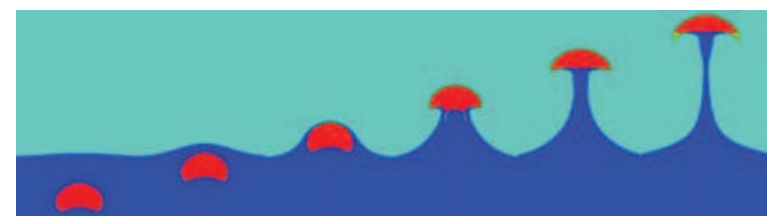

FIGURE 8. (Colour online) Evolution of the three-phase system for a bubble corresponding to $B o=52.9$ and $A r=11.7$ in series A. Comparison between $(a)$ the experimental sequence and $(b)$ computational predictions. The dimensionless time period between two consecutive images is $\sim 1.5$.
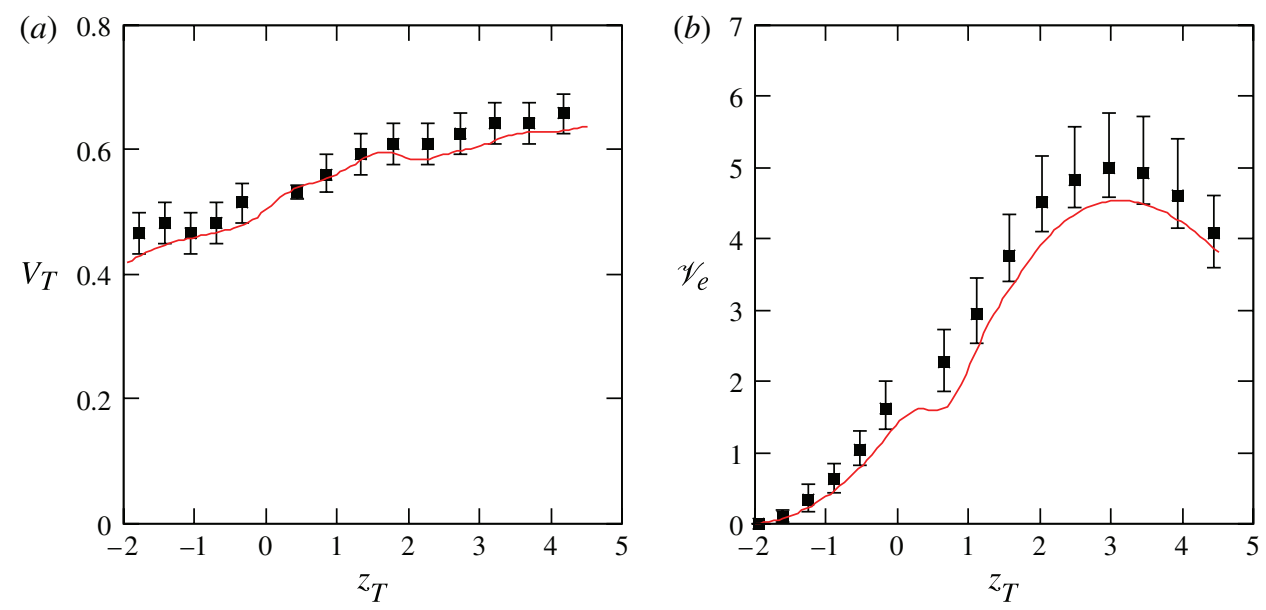

FIGURE 9. (Colour online) Evolution of ( $a$ ) the normalized rise speed $V_{T}$ and $(b)$ the displaced volume $\mathscr{V}_{e}$ for the bubble considered in figure 8 . See figure 5 for legend.

figure $7(a)$. The reason for this difference may easily be identified: while the bubble considered in the previous case is spheroidal (at least until it has totally emerged in the upper fluid), the present one exhibits an almost spherical cap shape throughout its rise. Therefore the two rise speeds follow drastically different laws. In the former case, the drag force directly depends on the fluid viscosity (for a given bubble aspect ratio) because dissipation is essentially generated in the bulk of the fluid since the flow about the bubble is unseparated. In contrast, the flow past a spherical cap bubble is massively separated at the back of the bubble and most of the dissipation takes place there. Thus the flow in the front region is close to irrotational and, provided the Archimedes number (and hence the Reynolds number) is large enough, the rise velocity of a spherical cap bubble is known to depend only on its radius of curvature $R^{*}$ in that region through the relation $V_{T}=(2 / 3) \mathscr{R}^{1 / 2}$, where $\mathscr{R}=R^{*} / d$ (Davies \& Taylor 1950). This result can actually be extended to account for finite viscous effects, assuming that the flow is viscous but still irrotational in the front region, yielding 


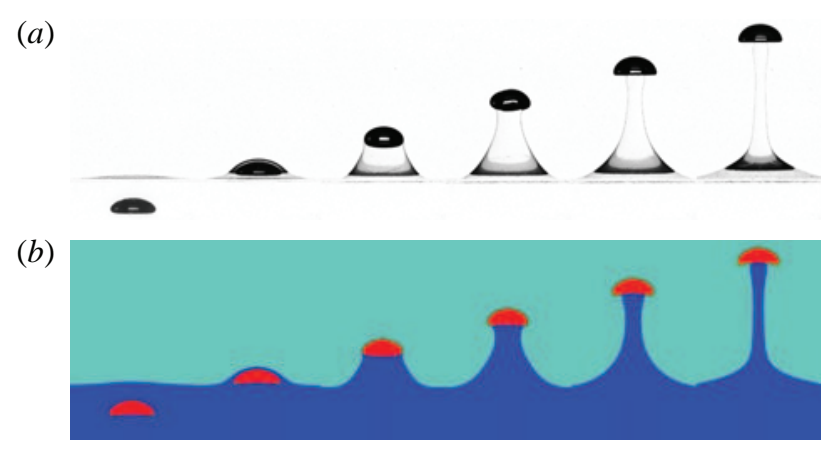

FIGURE 10. (Colour online) Evolution of the three-phase system for a bubble corresponding to $B o=29.5$ and $A r=42.6$ in the upper series B. Comparison between $(a)$ the experimental sequence and $(b)$ computational predictions. The dimensionless time period between two consecutive images is $\sim 1.7$.

(Joseph 2003)

$$
V_{T}=-\frac{4}{3 A r \mathscr{R}}+\frac{2}{3} \mathscr{R}^{1 / 2}\left\{1+\frac{4}{A r^{2} \mathscr{R}^{3}}\right\}^{1 / 2} .
$$

Given the above prediction, there is no reason for $V_{T}$ to change abruptly when the bubble emerges from the interface. Nevertheless, the secondary viscous corrections in (5.2) experience a jump due to the change in the value of the Archimedes number (from $A r$ to $A r_{u}$ ). In the present case, this jump makes viscous corrections negligibly small in the upper fluid, suggesting a small sudden increase of $V_{T}$ just after the bubble has crossed the interface. This may be the origin of the small bump visible in figure $9(a)$. The mild increase of $V_{T}$ as the bubble rises in the upper liquid also follows qualitatively the above prediction: as figure 8 shows, the bubble radius of curvature gradually increases (by $\sim 12 \%$ between the last two snapshots), which in turn results in a gradual increase of $V_{T}$. The rise speed is quantitatively well predicted by the above formulae: in the last snapshot $\mathscr{R} \approx 0.9$, yielding $V_{T} \approx 0.63$ according to (5.2), in close agreement with both experiment and computations. Not surprisingly, the displaced volume (figure $9 b$ ) reaches significantly larger values than in the previous case (figure $7 b$ ). The computations accurately capture the entire evolution of $\mathscr{V}_{e}$, although the small 'plateau' corresponding to the period during which the bubble crosses the interface seems somewhat exaggerated.

As in $\$ 5.2$, it is of interest to examine how a large difference in the viscosity contrast $\Lambda$ influences the evolution of bubbles belonging to the spherical cap family. For this purpose we consider a bubble with $B o=29.5$ and $A r=42.6$ in the upper series B. The corresponding evolution is displayed in figure 10. Since this bubble has a smaller Bond number compared to that of series A discussed above, it reaches the interface with a less dimpled shape and results in a spherical cap of smaller radius in the upper fluid. However, the similarity between the two shape evolutions is clear and is confirmed by the resemblances between the evolutions of the two rise speeds (figures $9 a$ and $11 a$ ).

The fact that the actual Archimedes number only decreases by $\sim 30 \%$ in the present case and remains moderate after the bubble has crossed the interface $\left(A r_{u} \approx 30\right)$, whereas it roughly increases by a factor of fifty in the previous case and becomes large $\left(A r_{u} \approx 500\right)$, has two main consequences. First, the column of entrained liquid 

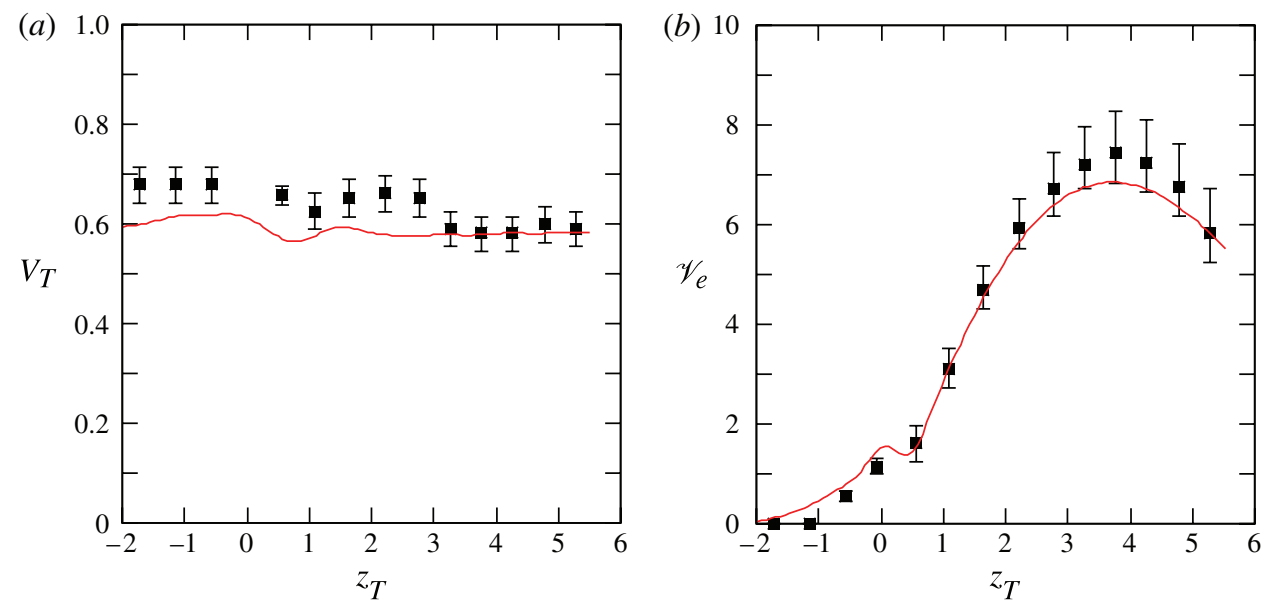

FIgURE 11. (Colour online) Evolution of ( $a$ ) the normalized rise speed $V_{T}$ and $(b)$ displaced volume $\mathscr{V}_{e}$ for the bubble considered in figure 10. See figure 5 for legend.

is somewhat thicker here (compare the end of the sequences in figures 8 and 10), yielding a maximum displaced volume (figure $11 b$ ) $\sim 50 \%$ larger. Second, the time required for the bubble to reach a stationary shape in the upper fluid is much shorter when $A r_{u}$ is moderate. This is why the rise velocity in figure 11(a) has already reached a steady value at the end of the sequence, while that in figure $9(a)$ is still increasing. The viscosity jump also influences the rise speed of spherical cap bubbles in an indirect and subtle manner. Indeed, the leading-order relation $V_{T} \propto \mathscr{R}^{1 / 2}$ implies $V_{T}^{2} \mathscr{S} \propto \mathscr{R}^{3}$, where $\mathscr{S}$ denotes the area of the bubble's horizontal crosssection. Therefore the balance of drag and buoyancy forces implies that, throughout the bubble ascent in each fluid, the variations of the bubble drag coefficient $C_{D}$ and those of its radius or curvature are linked by the condition $C_{D} \mathscr{R}^{3} \approx$ const. (there may be some variation of the cap angle, so $\mathscr{S} / \mathscr{R}^{2}$ may not be strictly constant). The drag coefficient of these bubbles is a decreasing function of the Archimedes number, being essentially determined by the viscous dissipation in the wake. Therefore when $A r$ jumps to $A r_{u}$ and the above constant jumps from 1 to $1-R$ after the bubble has crossed the interface, $\mathscr{R}$ has to increase or decrease, depending on whether $(1-R) C_{D}^{-1}\left(A r_{u}\right)$ is larger or smaller than $C_{D}^{-1}(A r)$, and the variation of $V_{T}$ follows. This implies that $\mathscr{R}$ and $V_{T}$ have to increase when $R$ is small and $\Lambda \ll 1$, while the density contrast forces them to decrease slightly when $\Lambda \approx 1$. These predictions may be verified in figures 8 and 9 (respectively figures 10 and 11), which correspond to the first (respectively second) scenario.

Note that, compared to the bubble of the lower series B examined in $\S 5.2$ (whose diameter is about two-thirds that of the present bubble), no sharp drop of the rise velocity is observed in figure 11 when the bubble approaches the horizontal interface. This is an indication that the capillary overpressure in the film that covers the bubble during this stage barely influences its dynamics, as will be discussed in more detail in the next section. The only discernible effect of this film is the small bump visible in the evolution of $V_{T}$ just before it reaches its final value: the corresponding video sequence indicates that this bump is associated with the late rupture of the film; a similar, albeit weaker and somewhat premature bump may also be discerned in the computations. The experimental/computational rise speed at the end of the sequence 
(a)

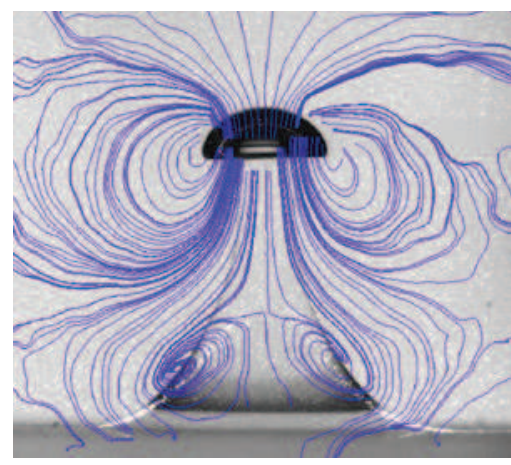

(c)

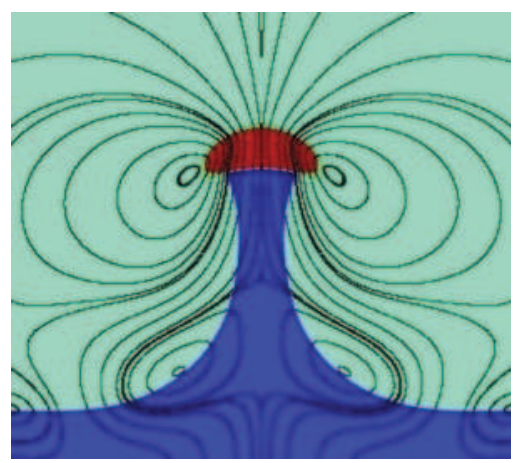

(b)

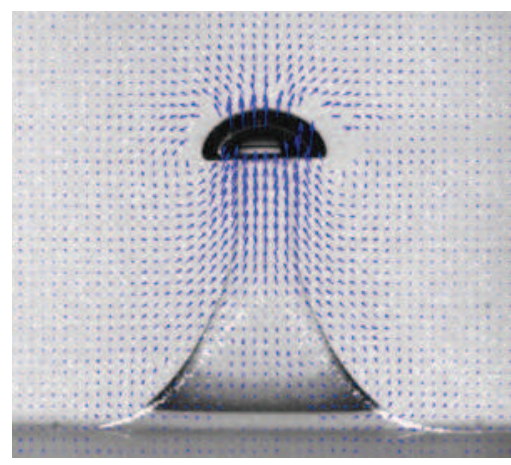

$(d)$

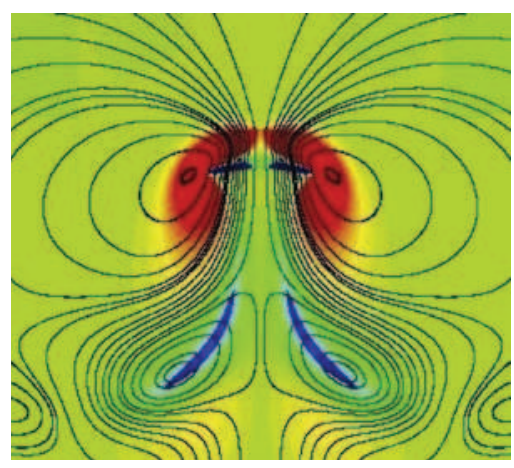

FIGURE 12. (Colour online) Flow pattern past the bubble considered in figure 10 at the time when its top stands at $z_{T} \approx 3.4$. $(a, b)$ Streamline and velocity fields obtained through PIV measurements; $(c, d)$ streamline and vorticity fields taken from computations. The azimuthal vorticity $\omega_{\theta}$ is normalized by $V_{T}(g / d)^{1 / 2}$. The red (respectively black/blue) regions correspond to positive (respectively negative) vorticity.

compares well with the inviscid prediction $V_{T}=(2 / 3) \mathscr{R}^{1 / 2}$ since the latter predicts $V_{T} \approx 0.58(\mathscr{R} \approx 0.75)$. Interestingly, the agreement with the prediction of (5.2) is not as $\operatorname{good}\left(V_{T} \approx 0.52\right)$, which may suggest that the assumption of viscous potential flow overestimates the influence of viscous effects on the rise speed. This is known to be the case with spherical bubbles for which the boundary layer resulting from the non-zero surface vorticity lowers the drag force and hence increases the rise speed (Moore 1963). Although the present result is obviously not sufficient to settle the matter definitely, it suggests that taking into account the normal viscous stresses (from which the viscous correction in (5.2) arises) without considering the shear-free condition responsible for the surface vorticity (which modifies the pressure distribution along the interface) may not be a suitable approximation.

Figure 12 displays PIV and computational determinations of the flow pattern around the bubble at the time when the entrained volume reaches its maximum. Not surprisingly, the upper half of the streamline pattern is dominated by a dipole structure associated with the bubble motion. While the upper part of the entrained column is still rising with the bubble, its lower part is already receding towards its initial position. The central part of the column is thus stretched axially, which creates the hyperbolic point visible on the column axis. At the same time the base of the column thickens and flows radially on the horizontal interface, which induces an axial compression and results in another hyperbolic point near the intersection of 
the column axis with the plane of the undisturbed interface. Positive values of the azimuthal vorticity $\omega_{\theta}$ are of course concentrated close to the bubble surface, with a dimensionless maximum $\omega_{\max } \approx 23.1$ located slightly ahead of the region of maximum curvature where most of the vorticity is produced. A small, nearly horizontal and almost black zone can be discerned just at the back of the bubble, in the region where its surface is concave. This corresponds to negative vorticity (with a minimum $\left.\omega_{\min } \approx-7.2\right)$ generated in the attached eddy, where the tangential velocity goes from the bubble axis to the region of maximum curvature. Finally a thin shear layer having negative vorticity (with $\omega_{\min } \approx-1.6$ ) is observed along the fluid-fluid interface in the lower part of the column; this structure is of course a direct consequence of the entrainment of the outer fluid, which has low downward velocities, by the heavy fluid which recedes faster.

\subsection{A toroidal bubble}

We finally consider the situation corresponding to $B o=48.2$ and $A r=7840$ in series $\mathrm{C}$, which, according to our observations, results in a toroidal bubble. Indeed, when viscous effects are small enough, large initially nearly spherical bubbles are known to undergo a topological change and become toroidal. The basic mechanism that drives this transition is the growth of the tongue that forms at the bottom of the bubble, owing to the hydrostatic pressure difference between the top and bottom regions. As it develops, this tongue dramatically reduces the height of the bubble along its axis and quickly leads to pinch-off unless capillary forces are strong enough to limit its development. Bonometti \& Magnaudet (2006) showed that, when $\mathrm{Ar}>10^{3}$, this topological transition always happens with initially spherical bubbles whose Bond number is beyond a critical value in the range $32-35$. Therefore the case considered here is supercritical and the bubble switches from its initial shape to the toroidal configuration well before it reaches the fluid-fluid interface. Toroidal bubbles rising in low-viscosity fluids have been studied experimentally (Walters \& Davidson 1963) and theoretically (Pedley 1968), the bubble then being considered as a hollow vortex ring. Given the large values of $A r$ generally associated with such bubbles, their dynamics are essentially inviscid during most of their lifetime. Provided the core has a circular cross-section, the evolution of the dimensionless ring radius $R(t)$ and rise velocity $V(t)$ is then governed by

$$
\frac{R(t)}{R_{0}}=\left(1+\frac{t-t_{0}}{6 \Gamma R_{0}^{2}}\right)^{1 / 2}, \quad V(t)=\frac{\Gamma}{4 \pi R}\left(\log 16\left(3 \pi R^{3}\right)^{1 / 2}-\frac{1}{2}\right),
$$

where $R_{0}=R\left(t_{0}\right)$ and the circulation $\Gamma$ around the core has been normalized by $\left(g d^{3}\right)^{1 / 2}$. The core radius $a(t)$ may then be determined thanks to the condition of volume conservation $12 \pi R(t) a^{2}(t)=1$. Toroidal bubbles rising in inviscid fluid have been investigated computationally by Lundgren \& Mansour (1991) using a BIM, well before the full Navier-Stokes computations of Bonometti \& Magnaudet (2006). Their results confirmed inviscid predictions and revealed the existence of significant oscillations of the ring radius and core shape when the ratio of the ring-to-core radii is small enough.

In the present context, as figure 13 shows, the most salient feature revealed by experiments as well as by computations is that the bubble remains encapsulated in a thick body of heavy fluid after having crossed the original position of the interface. This is due to the circulation around the bubble core which induces an upward fluid velocity in the central part of the ring (as may be discerned in figure 14), which in 
(a)

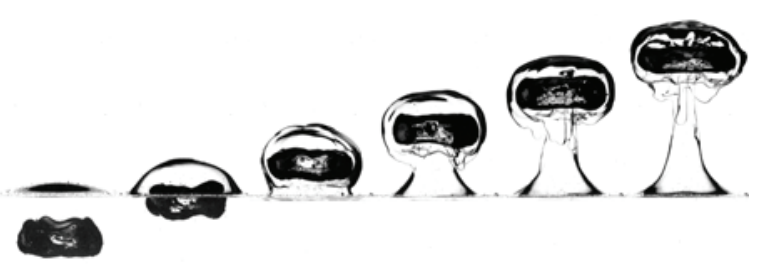

(b)

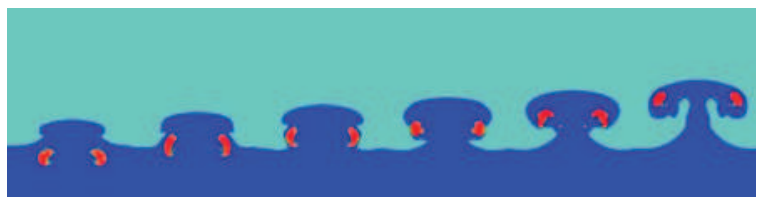

(c)

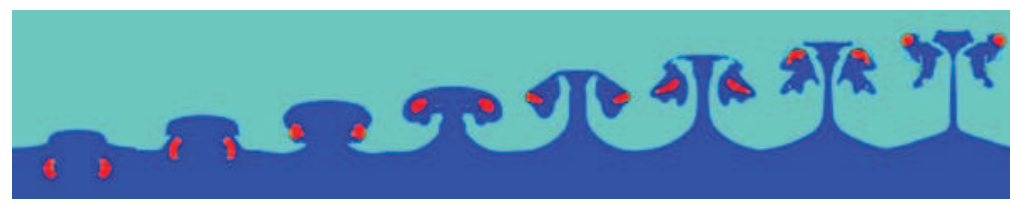

FIGURE 13. (Colour online) Evolution of the three-phase system for a bubble corresponding to $B o=48.2$ and $A r=7840$ in series C. (a) Experimental sequence and $(b, c)$ computational predictions. The dimensionless time period between two consecutive images is $\sim 0.66$ in the upper two rows and $\sim 1.25$ in the bottom row.
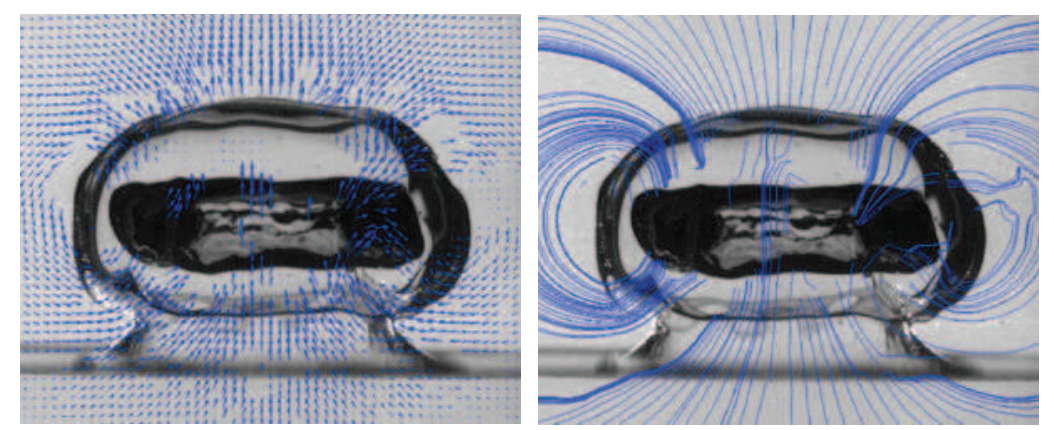

FIGURE 14. (Colour online) Experimental velocity and streamline patterns past a $6 \mathrm{~cm}^{3}(d \approx$ $22.5 \mathrm{~mm}$ ) toroidal bubble just after it has crossed the position of the undisturbed interface.

turn tends to 'feed' the film ahead of the bubble with fresh heavy fluid withdrawn from the entrained column. Note that, according to the computational sequences, the core is far from circular and undergoes strong oscillations, in line with the findings of Lundgren and Mansour.

Unfortunately, comparison of present experimental results with theoretical and computational predictions for $R(t)$ and $V(t)$ can hardly be made quantitative, for several reasons. First, it must be kept in mind that the original bubble is injected much closer to the undisturbed interface in the computation than in the experiment. Moreover, given the design of the injection system, this original bubble is certainly not spherical in the latter, and this has a direct influence on the time (i.e. height) at which the topological transition takes place as well as on the value of the circulation. Indeed Bonometti \& Magnaudet (2006) showed that under certain conditions, bubbles with 

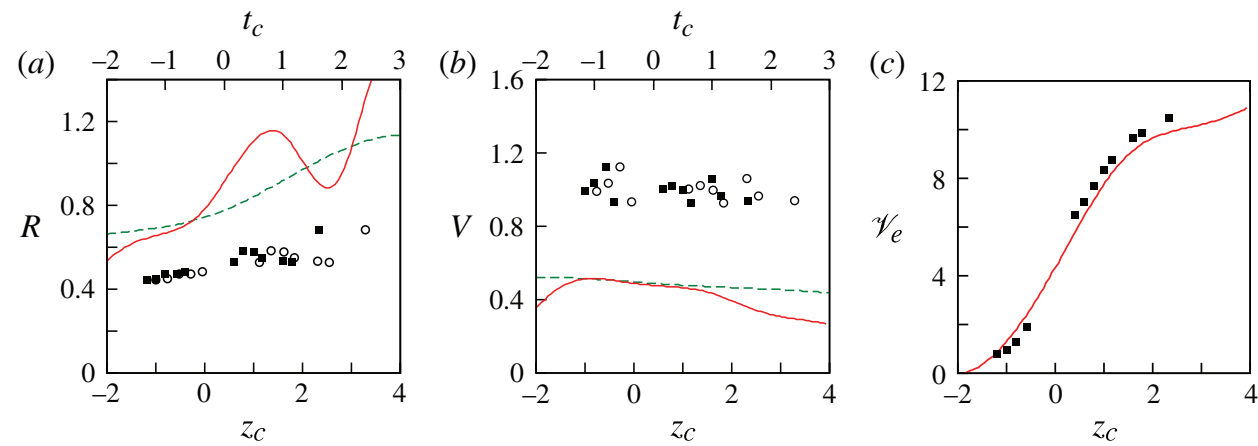

FIGURE 15. (Colour online) Evolution of $(a)$ the normalized ring radius $R,(b)$ rise speed $V$, and $(c)$ entrained volume $\mathscr{V}_{e}$ for the toroidal bubble displayed in figure 13 versus the dimensionless vertical position $z_{c}$ of the bubble's centre of inertia above the undisturbed interface (black squares and solid line). Subfigures $(a)$ and $(b)$ also display the corresponding evolutions versus the dimensionless time $t_{c}$, with $t_{c}=0$ when $z_{c}=0$ (open circles and dotted line). The 'experimental' ring and core radii are obtained by evaluating the dimensionless area $S^{*}$ and perimeter $P^{*}$ of the projected surface $\mathscr{S}$ of the bubble on images such as that of figure 14, and equating these quantities with their counterparts for a torus with a circular core, which yields the conditions $S^{*}=4 R a+\pi a^{2}$ and $P^{*}=4 R+2 \pi a$; the rise velocity is defined as the time variation of the vertical position of the centre of inertia of $\mathscr{S}$ and the entrained volume is obtained by fitting the fluid-fluid interface contour with a curve preserving the left-right symmetry, evaluating the enclosed volume by invoking axisymmetry and removing the known bubble volume; this procedure does not work when the bubble crosses the undisturbed position of the interface, which is why no data appear in $(c)$ during that stage. The computational ring radius is defined as $R(t)=\int r^{2} C(r, z, t) \mathrm{d} r \mathrm{~d} z / \int r C(r, z, t) \mathrm{d} r \mathrm{~d} z$, where $C$ is the local volume fraction of air and integration is performed over the whole computational domain; the rise velocity is defined as $V(t)=\int r U_{z}(r, z, t) C(r, z, t) \mathrm{d} r \mathrm{~d} z / \int r C(r, z, t) \mathrm{d} r \mathrm{~d} z$, where $U_{z}$ is the local vertical fluid velocity.

a large enough initial oblateness can even preserve a spherical cap shape throughout their life, whereas spherical bubbles with the same volume quickly become toroidal Because of these two factors, there is no chance that real and computed bubbles of a given volume give birth to a toroidal bubble at the same distance from the interface. Therefore, the corresponding two toroidal bubbles do not have the same 'age' when they reach the position of the undisturbed interface, nor do they probably have the same initial ring radius $R_{0}$ and circulation $\Gamma$. Also, given the large value of the Archimedes number, the actual bubble shape and the flow about it are threedimensional (with significant azimuthal fluctuations as may be seen in figure 14), whereas available predictions and present computations assume an axisymmetric evolution.

Having pointed out these issues, we are left with the possibility of performing qualitative comparisons. Figure 15 displays the evolution of the ring radius $R$, rise velocity $V$ and entrained volume $\mathscr{V}_{e}$; the way these quantities are defined and extracted from the complex bubble and interface shapes revealed by figure 13 is detailed in the corresponding caption. Figure 15(b) shows that the rise velocity is a slowly decreasing function of time and vertical position, a trend that was to be expected owing to the $R^{-1} \log R$ term in the second of (5.3). The experimental and computational evolutions are qualitatively similar; during the late stages, say for $1.5<z_{c}<2.5$ (here $z_{c}$ is 
the normalized vertical position of the bubble centre of inertia above the undisturbed interface), the computation predicts a more pronounced decrease of $V$ which, as will be seen below, is related to the presence of the body of heavy fluid that moves with the bubble. Nevertheless the magnitude of $V$ is roughly twice as large in the experiments, suggesting a higher value of the circulation due to the difference in the bubble shape at the time of pinch-off. Indeed, starting from a spherical bubble, Lundgren \& Mansour found $\Gamma \approx 1.5$ for $B o=40$, and this value is certainly close to that corresponding to $B o=48.2$. When the computed bubble crosses the position $z_{c}=-1$, figure $15(a)$ indicates $R=0.65$, which, by setting $\Gamma=1.5$ in the second of (5.3), yields $V=0.505$, in excellent agreement with the computational prediction $V=0.51$ reported in figure $15(b)$. At the same position, assuming the same value of $\Gamma$, the measured ring radius $(R=0.45)$ would lead to $V=0.58$ instead of $V \approx 1.0$ as found experimentally. This strongly suggests that the difference in magnitude between the experimental and computational rise speeds essentially results from differences at the instant in time when the toroidal bubble is formed.

At first glance, the experimental and computed evolutions of the ring radius versus $z_{c}$ reported in figure 15(a) display contrasting trends. However, it must be kept in mind that, owing to its nearly twice as small rise speed, it takes typically twice as long for the computed bubble to cross a given vertical distance (compare the final two positions in figure $13 a, b$ ). This is why the evolution of $R$ versus time is more revealing. Using this representation, it can be seen that the growth of the ring radius follows qualitatively similar trends in both evolutions up to the end of the experimental observations. Selecting again $\Gamma=1.5$ and $t_{0}=-1$, i.e. $R_{0}=0.70$ according to the computations, the first of (5.3) predicts $R\left(t_{c}=0\right)=0.775$ which is very close to the computational prediction $R\left(t_{c}=0\right)=0.78$. This allows us to conclude that the early evolution of the computed bubble is in line with theoretical predictions. The late evolution of the ring radius (beyond the end of the experimental observations) is more complex, since $R$ is seen to decrease for some time and then increase again. These variations are better understood by considering the whole computational sequence displayed in figure $13(c)$. As revealed by this sequence, owing to the large volume of heavy fluid entrained by the bubble, a compound toroidal body made of the bubble plus a large fraction of the top of the entrained column progressively forms and starts moving autonomously in the upper fluid (note that the top of the column is no longer connected to this toroidal body at the end of the sequence). Within this compound body, which may be seen as a nearly neutrally buoyant vortex ring, fluid particles rotate in the direction given by $\Gamma$. Therefore, in addition to its own motion, the bubble core undergoes some entrainment within this body of heavy fluid: it first moves outward (which provides an extra increase in $R$ clearly visible in the fourth and fifth snapshots), then inward and downward (which briefly reduces $R$ in the sixth snapshot and lowers the rise speed as seen in the range $1.5<z_{c}<2.5$ in subfigure $b$ ), then outward and upward again. Unfortunately, the experimental sequence is not long enough to reach similar times and determine whether the same phenomenon occurs or is rather due to the axisymmetry imposed in the computations.

Since the bubble remains encapsulated in the film throughout the period of observation, the evolution of the entrained volume (figure $15 c$ ) is remarkably smooth (compare with figure $11 b$ for instance). In line with the 'feeding' mechanism provided by the circulation around the core, its maximum is larger and takes place at a greater distance from the undisturbed interface than in all other configurations. Surprisingly, the experimental and computational evolutions of $\mathscr{V}_{e}$ versus $z_{c}$ are in remarkable agreement. This may be totally fortuitous, but we believe that, for toroidal bubbles, 


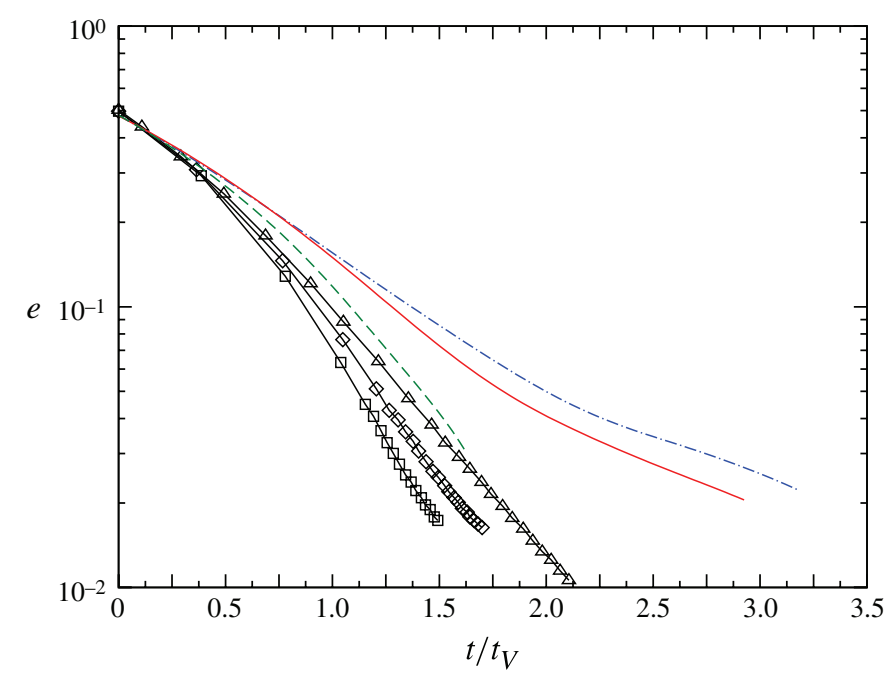

FIGURE 16. (Colour online) Evolution of the normalized film thickness $e$ computed for the three spheroidal bubbles considered in $\S 5$ (semi-log coordinates). Time is normalized by the viscous scale $t_{V}=12 \mu_{1} /\left(\rho_{1} g d\right)=12 A r^{-1}(d / g)^{1 / 2}$; its origin is taken at the instant when the dimensional film thickness is $d / 2$. Solid line, situation corresponding to figure $3\left(B o=3.0, B_{I}=4.9, \Lambda=1.11\right)$; dash-dotted line, situation corresponding to figure $4\left(B o=13.2, B_{I}=21.3, \Lambda=0.96\right)$; dotted line, situation corresponding to figure 6 $\left(B o=13.3, B_{I}=21.6, \Lambda=0.0175\right)$. The lines with symbols are the results of Pigeonneau \& Sellier (2011) for a bubble approaching a free surface $(R=1, I=1, \Lambda=0): \square$, $B o=B_{I}=3.6 ; \diamond, B o=B_{I}=6.0 ; \triangle, B o=B_{I} \geqslant 12.0$.

the entrained volume depends primarily on the bubble volume rather than on the rise velocity and detailed geometry of the bubble. Further comparisons between experiments and computations for other bubble volumes are required to assess this conjecture.

\section{Film drainage and tailing configurations: the role of the interfacial Bond number and the viscosity and density ratios}

Little has been said up to now regarding the role of the relative interfacial tension $I$ or better that of the interfacial Bond number $B_{I}=B o / I$. It is the purpose of this section to re-examine some of the material discussed in $\S 5$ in the light of results available in the literature and of a simple model developed in appendix D to better understand how this parameter, jointly with the density and viscosity ratios $R$ and $\Lambda$, influences the dynamics of the system when the bubble gets close to the undisturbed position of the interface.

Let us first consider the influence of interfacial and viscous effects on the drainage of the film that forms ahead of the bubble when it reaches the interface. In the low-Reynolds-number limit, the various computational studies based on the BIM (Chi \& Leal 1989; Manga \& Stone 1995; Pigeonneau \& Sellier 2011) consistently show that increasing $B o$ and $B_{I}$ up to $O(10)$ values slows down the film drainage, while no significant influence of interfacial forces is observed for larger values. Indeed, increasing $B o$ and $B_{I}$ allows the two boundaries of the film to deform more easily, causing the film thickness to remain more uniform during the drainage process. This in turn increases the area of the small-gap region and thus slows down the drainage 
since the body force available to squeeze the entire film is fixed. Figure 16 shows the evolution of the film thickness $e$ (measured on the vertical axis and normalized by $d / 2$ ) predicted by the computations for the three spheroidal bubbles considered in $\S 5.2$ which all correspond either to $\Lambda=O(1)$ or to $\Lambda \ll 1$. Although the lower part of the curves (say, that corresponding to $e<4 \times 10^{-2}$ ) is certainly contaminated by numerical inaccuracies and must be disregarded, the general trends observed for larger gaps are clear. First it is worth noting that, for $t / t_{V}>1$, i.e. times larger than the viscous time scale $t_{V}=12 A r^{-1}(d / g)^{1 / 2}$, figure 16 essentially confirms the exponential evolution of the film thickness already observed in presence of a free surface (Debrégeas, de Gennes \& Brochard-Wyart 1998). As this exponential behaviour directly results from the plug velocity profile in the film, while a parabolic profile is known to produce a power-law decay (Jones \& Wilson 1978), present results suggest that the velocity profile is still mostly of plug type for $O(1)$ values of $\Lambda$.

Among the two situations involving $O(1)$ viscosity ratios (solid and dash-dotted lines in figure 16), the smaller $B_{I}$ the faster the drainage, in agreement with previous low-Reynolds-number findings. In those two cases, the drainage is found to be significantly slower than in the free-surface situations recently considered by Pigeonneau \& Sellier (2011), as shown by comparing the solid line with the squares $\left(B_{I} \approx 3\right.$ in both cases) and the dash-dotted line with the triangles $\left(B_{I} \geqslant 12\right.$ in both cases). As this trend is observed for any $B_{I}$, we can conclude that the difference is due to the viscosity ratio: the smaller $\Lambda$, the more efficient the drainage since the stretching of the upper film surface does not induce any significant stress (hence no dissipation) when $\Lambda \ll 1$. This is also confirmed by comparing the dash-dotted and dotted line which correspond to nearly identical values of $B_{I}$ but markedly different values of $\Lambda$ : the film is drained much faster when $\Lambda \ll 1$ and its evolution is then close to that predicted by Pigeonneau \& Sellier in the same range of Bond numbers. However, it may be noticed that the corresponding drainage is still slightly slower than at a free surface (dotted line versus triangles). This is in line with the above argument regarding the spatial variation of the film thickness: as $I=1$ at a free surface while $I \approx 0.6$ here, the upper film surface deforms somewhat more easily for a given $B o$ in the present case, so that some more energy is required to drain the entire film region. To conclude, the computational results displayed in figure 16 confirm that low-Reynolds conclusions are still valid in the inertial regime: the smaller $B_{I}$ the faster the film drainage for a given $\Lambda$; the smaller $\Lambda$ the faster the film drainage for a given $B_{I}$ (the figure may give the impression that the influence of the viscosity ratio is dominant but it must be kept in mind that $\Lambda$ varies by a factor of 60 among the three curves while $B_{I}$ only varies by a factor of 4 ).

Let us now discuss how effects of interfacial tension and density contrast influence the decrease of the bubble rise speed and residence time near the interface. This is most easily achieved by relying on a model predicting how the net buoyancy force acting on the bubble is altered when the latter stands at a given dimensionless altitude $\mathfrak{h}$ above the undisturbed position of the interface. We develop such a model in appendix D by considering how the joint presence of a non-zero interfacial tension and of an entrained column of heavy fluid influence the static pressure distribution at the surface of a spherical bubble. This model provides the distribution of the effective body force $g_{e}$ as a function of $\mathfrak{h}$ and shows that it goes through a minimum, $g_{m}$, given by (D 2) or (D 3) in the two limits $R B_{I} \rightarrow 0$ or $R B_{I} \rightarrow \infty$, respectively; it is of interest to notice that requiring $g_{m}>0$ in the latter yields the criterion derived by Greene $e t$ al. (1988). Comparing (D 2) and (D 3), this model shows that the major two roles of the interfacial tension are to increase the pressure in the film ahead of the bubble and the 
volume of heavy fluid it displaces. Hence, for a given density contrast $R$, the larger $B_{I}$ the smaller the decrease of the effective body force when the bubble is close to the undisturbed interface. This is why in all configurations we considered, increasing $B_{I}$ results in a reduction of the slowing down of the bubble rise near the fluid-fluid interface, although it actually slows down the film drainage, as we saw above. Also, increasing the density contrast between the two fluids makes the entrained column of heavy fluid decrease the net buoyancy acting on the bubble; this effect is frequently referred to as a 'drag increase' (Geller et al. 1986; Srdić-Mitrović et al. 1999) but it must be kept in mind that, in contrast with the usual origin of drag in a homogeneous fluid, it has nothing to do with vorticity generation at the body surface. Hence, as (D 2) and (D 3) show, the larger $R$ the most severe the slowing down of the bubble near the undisturbed position of the interface.

Considering the above findings, two different scenarios may happen. If $g_{e}$ vanishes at some critical altitude $\mathfrak{h}_{0}$, the bubble stops there and cannot rise any more until the drainage has been completed and the film has eventually ruptured under the effect of long-range forces. This is what happens in the 'film-drainage' configuration discussed in $\$ 5.1$. In contrast, if $g_{e}$ remains positive for any $\mathfrak{h}$, the bubble may go on rising while the film around it is not yet totally drained (this corresponds to all situations discussed in $\S \S 5.2$ and 5.3). In that case, the bubble ascent is only briefly slowed down by the decrease of $g_{e}$ in a certain range of $\mathfrak{h}$ and its residence time near the undisturbed position of the interface is generally much shorter than that required to drain the film. A column of heavy fluid develops behind the bubble as $\mathfrak{h}$ increases, a distinctive feature that led Geller et al. (1986) to term this configuration 'tailing'. The film may ultimately break ahead of the bubble or some hydrodynamic mechanism (such as viscous stretching or an instability) may neck down the column earlier, its upper part then continuing to rise with the bubble while the lower part recedes toward the initial position of the interface (as in the late stages of figures 8 and 10).

The first two situations considered in $\S 5$ illustrate these two scenarios and offer an opportunity to check the predictions of the model established in appendix D. Given the physical parameters corresponding to the situation considered in $\S 5.1$, (D 1) predicts $g_{e}$ will vanish for $\mathfrak{h}_{0} \approx 0.5$, which is in good qualitative agreement with the experimental observations reported in figure 3 (the agreement cannot be fully quantitative since bubble deformation was found to be large in that case and is totally neglected in the model). If we rather consider the smallest bubble examined in $\S 5.2$ (figure 4 ), the model yields $g_{m} / g \approx 0.38$ for $\mathfrak{h}=\mathfrak{h}_{m} \approx 1.3$. Since $\Lambda$ is close to unity in that case, one can roughly consider that the bubble rises in a homogeneous viscous fluid and estimate its minimum rise speed $V_{m}$ by using (5.1) in which we replace $A r$ by $\left(g_{m} / g\right) A r$. This yields $V_{m} \approx 0.115$ for $\mathfrak{h}_{c} \approx 1.3$, a prediction which compares well with the magnitude and position of the minimum rise speed observed in figure 5 .

To conclude this section, we wish to stress again the two independent and apparently antagonistic effects of the interfacial Bond number enlightened by present results. On the one hand, increasing $B_{I}$ increases the energy required to drain the film ahead of the bubble, thus lowering the drainage process. On the other hand, increasing $B_{I}$ lowers the overpressure in the film and thus reduces the slowing down of the bubble near the fluid-fluid interface, thus allowing it to rise more easily above the initial position of this interface. This is why, for large enough $B_{I}$, the overall evolution of the bubble may show a bare macroscopic influence of the interface (essentially visible for some time through the entrained column of heavy fluid), although at a microscopic scale interfacial effects may be at play for a long time, owing to the slow viscous drainage of the film. 


\section{Summary and concluding remarks}

In this paper we considered the inertial dynamics of a single bubble that rises across an initially horizontal interface separating two Newtonian fluids. By varying the bubble diameter and the viscosity contrast over a wide range, we were able to study several aspects of this phenomenon for almost all possible bubble shapes (from spherical to toroidal) and various scenarios of interface crossing. As reviewed in $\S 1$, most available studies of that problem, both experimental and computational, focused on creeping flow conditions and essentially considered situations corresponding to the film-drainage configuration, with the noticeable exception of Maru et al. (1971) and Geller et al. (1986). The only two 'modern' studies of similar gas/liquid/liquid systems with non-zero inertia known to us (Kemiha et al. 2007; Dietrich et al. 2008) spanned much more limited conditions: the former focused on spheroidal bubbles with diameters in the range $3.5-5 \mathrm{~mm}$ and a single couple of Newtonian fluids with $\Lambda \approx 0.15$, while the latter considered bubbles with diameters in the range $7-10 \mathrm{~mm}$ and viscosity ratios less than 0.5 . Moreover, in these studies emphasis was put on global quantities such as the 'residence time' at the interface, rather than on the elementary physical mechanisms at play. This is why we think the present investigation is the first to consider in detail the respective roles of inertial, viscous and capillary effects in the evolution of this system over a broad region of the $(A r, B o, \Lambda)$ parameter subspace. Also, very few direct simulations of that system with non-zero inertia were reported in the past. Kemiha et al. (2007) employed an Eulerian approach qualitatively similar to that used here with spheroidal bubbles corresponding to the regime illustrated in figure 4. However, owing to the coarse grid and narrow computational domain they used, their results had low accuracy and could only be qualitatively compared with experimental data. Therefore, we regard the computational exploration and the systematic comparison with the experimental evolutions provided in $\S 5$ as a significant step forward in the use of direct numerical simulation for investigating the basic physical processes at work in three-phase flows.

In the rest of this section, considering successively physical processes and computational issues, we summarize the main findings of the present investigation and indicate some directions for future work.

\subsection{Dynamics of the three-phase system}

As $\S 5$ showed, the dynamic features associated with the interface crossing sequence turn out to be markedly different with the various types of bubbles. In particular, for values of $B_{I}=B o / I$ in the range $1-10$, small spheroidal bubbles are first severely slowed down or even stopped by the overpressure that sets in within the film as they get close to the interface, while spherical cap bubbles (which correspond to much larger $B_{I}$ ) are barely affected by the existence of this film. Also, the smaller the viscosity of the upper fluid (i.e. $\Lambda$ ), the smaller the influence of the film on the overall dynamics, as revealed for instance by the comparison between results of figures 4 and 6, two cases with almost similar values of $B o$ and $A r$. This is essentially because decreasing this viscosity makes the upper boundary of the film more mobile, as figure 16 confirms.

Another important point highlighted by the present investigation is the strong connection between the evolution of the bubble rise speed and the readjustment of its shape to the new conditions it encounters as it starts rising in the upper fluid. Present experiments all correspond to $S<1$, so that the actual Weber number is larger in the upper fluid, making the bubble deformation easier. This is why, for spheroidal bubbles, this readjustment makes the evolution of the rise speed much milder than it would 
be if the bubble shape had remained unchanged. Hence, for instance, the intuitive idea that the rise speed of such bubbles in the upper fluid should be roughly $\Lambda^{-1}$ times that in the lower fluid is generally grossly in error, as illustrated by figures 6 and 7. In contrast, the rise velocity of spherical cap bubbles evolves as $\mathscr{R}^{1 / 2}$, which forces the drag coefficient, itself a decreasing function of the Archimedes number, to evolve roughly as $\mathscr{R}^{-3}$. This is why, when the bubble enters the upper fluid and the Archimedes number jumps from $\mathrm{Ar}$ to $A r_{u}$, its radius of curvature and rise speed are both seen to increase when $\Lambda \ll 1$ (figures 8 and 9), while they slightly decrease when $\Lambda=O(1)$ (figures 10 and 11).

A central feature of the three-phase system is the column of heavy fluid that forms and is entrained behind the bubble when the flow conditions allow it to rise above the initial position of the interface. The entrained volume increases until the bubble has travelled a few diameters above that position. Its maximum is significantly larger with spherical cap bubbles than with spheroidal bubbles as, for a given volume, the former offer a larger cross-section than the latter at the time the bubble crosses the interface. The viscosity contrast has a large influence on the development and shape of the column for at least two reasons. First, depending on whether $\Lambda$ is small or large, it creates extensional or compressional stresses along the column; when the former are large enough, a gas tail resulting eventually in secondary bubbles may form, as in figure 6. Second, a large (respectively small) $\Lambda$ implies that the relative magnitude of inertial and viscous effects decreases (respectively increases), leading to a thicker (respectively thinner) column, as the comparison of figures 8 and 10 clearly shows. Toroidal bubbles correspond to a specific situation because the film of heavy liquid ahead of the bubble is fed by the entrained column through the central part of the ring, so that the bubble remains encapsulated in a thick film.

The density contrast between the two fluids is moderate in the systems we considered and its most visible effect is the slight reduction experienced by the bubble rise velocity in the upper fluid when $\Lambda=O(1)$. However, when the bubble reaches the fluid-fluid interface, this parameter plays an important role in the film-drainage/tailing transition, as the discussion in $\S 6$ and the model developed in appendix D show. This is because the volume of heavy fluid displaced by the bubble exceeds its own volume, owing to the development of the entrained column. Large density contrasts are encountered in applications, especially with liquid metal as the heavy phase (e.g. $R \approx 0.93$ for the mercury/water system). In such systems of fluids, only the film-drainage configuration is possible if the dimensionless displaced volume $\mathscr{V}_{H}$ (defined in figure 20 ) approaches $1 / R \approx 1$. According to (D2), $\mathscr{V}_{H}$ may reach values significantly larger than unity for small-to-moderate interfacial Bond numbers, so that only bubbles with very large $B_{I}$ may result in a tailing configuration in such systems of fluids. This is why bubbles with Bond numbers of several tens, i.e. spherical cap bubbles, may then remain trapped at the interface, as was observed by Cranga et al. (2001).

Finally, it is important to realize that the present study mostly focused on the transient phenomena that affect the three-phase system when the bubble is not far from the fluid-fluid interface. In certain cases, especially with small bubbles, no mark of the passage across the interface subsists after the bubble has risen a few diameters in the upper fluid. However, this may not be true with larger bubbles, which may continue to tow a significant volume of heavy fluid in the form of a single attached drop or of a string of droplets. This permanent entrainment phenomenon, which is important in applications such as iron processing, was studied by Greene et al. (1988) and Greene, Chen \& Conlin (1991) who derived simple criteria to predict its threshold 
and the associated permanently entrained volume $\mathscr{V}_{P}$. It could not be studied here, owing to the limited height of the upper fluid layer (and in some cases of the field of view). Nevertheless this is an aspect deserving future investigation, especially because available models attempt to predict $\mathscr{V}_{P}$ on the sole basis of static force balances, and their predictions frequently exceed the observed entrained volumes by one to two orders of magnitude. Indeed, Greene et al. (1991) noticed that the viscosities of both fluids had a significant influence on the values of $\mathscr{V}_{P}$ they determined, which is no surprise when one considers dynamical features such as the viscous stretching of the entrained column clearly visible in figure 8 . Thus there is a clear need to improve the understanding and modelling of the tearing mechanisms acting on the entrained column, so as to properly incorporate the main features of the dynamics in predictive models.

\subsection{Strengths and current limitations of Eulerian computational approaches}

As the various examples discussed in $\S 5$ showed, computational approaches based on the full Navier-Stokes equations are able to reproduce quantitatively most aspects of the dynamics of inertial gas/liquid/liquid systems. In particular, the detailed evolutions of the bubble shape, rise speed, volume and shape of the entrained column are generally well predicted, although some flow details are not properly captured owing to the limitations inherent in the $O\left(10^{-2} d\right)$ grid resolution we selected. Moreover, some of the material used in $\S \S 5$ and 6 was provided by the computations and could not have been obtained experimentally, given the limitations of the optical technique and the device we used. This is for instance the case of the position, shape and strength of the thin vortical layers displayed in figure 12, of the detailed dynamics of the core of the toroidal bubble (figure 13) or of the evolution of the film thickness displayed in figure 16 .

The only clear failure of the computational predictions we observed occurred in the situation where a small bubble with $B o=O(1)$ remains trapped at the fluid-fluid interface for a very long time, owing to the slow drainage of the film. Some bias has also been noticed with bubbles that spend a significant, albeit shorter time at the interface: in such cases the acceleration experienced by the bubble when it starts rising in the upper fluid occurs somewhat too early, owing to the premature film rupture. These observations make it clear that the major current shortcoming of this type of approach is its inability to predict correctly the dynamics of very thin films in situations where they control the evolution of the system. As in every one-fluid formulation (such as volume of fluid, level set or front tracking approaches), numerical pinch-off occurs sooner or later when the film thickness becomes less than a few grid cells because the solution of (3.1) suffers unavoidably from some numerical diffusion and, as discussed in appendix $\mathrm{C}$, the finite thickness of the numerical interfaces results in a poor evaluation of the physical properties within the film and in contamination of the local flow dynamics by spurious capillary effects. Obviously, this numerical pinchoff can be delayed by increasing the grid resolution locally, but even this technique experiences practical limitations when the film thickness becomes several orders of magnitude less than the dominant flow scales. This problem is now recognized as being one - if not the - major limitation of current direct numerical simulation (DNS) approaches of multiphase flows involving drop or bubble suspensions and possibly solid walls, since very thin inter-particle and wall-particle films are ubiquitous in such flows. Remedies other than local dynamic grid refinement (e.g. Popinet 2003, 2009) are still in their infancy. A recent attempt (Thomas, Esmaeeli \& Tryggvason 2010) explores the possibility of computing the motion and deformation of drops sliding 
along a wall by combining DNS in the bulk with the solution of an additional pair of one-dimensional equations governing the evolution of the local thickness and maximum velocity in the film separating the drop from the wall, respectively. This kind of 'subgrid scale' approach is very promising. However, it takes advantage of the fact that the shape and position of the substrate on which the film develops are known in advance. In the situations considered here this is by no means the case because the film characteristics and location depend on the flow history. This is why we plan to devote future efforts to extending such approaches to curved films whose position and mean shape are an intrinsic part of the solution.

\section{Acknowledgements}

We thank G. Ehses for his essential role in the design of the experiment and S. Cazin for his expert technical assistance with the optical and high-speed imaging acquisition systems. The computations performed with JADIM owe much to A. Pédrono, who is at the heart of the collaborative work carried out around this code. This work was funded by IRSN whose financial support is greatly appreciated. This work was granted access to the HPC resources of CALMIP under the allocation 2011-P1142.

\section{Appendix A. Extension of the CSF model to three-phase systems}

The expression of the capillary force $\boldsymbol{T}$ in (3.5) deserves some discussion. Computational approaches of two-phase flows involving the transport of the volume fraction of one of the fluids frequently make use of the CSF formulation first proposed by Brackbill et al. (1992) and express the capillary force per unit volume in the form $\boldsymbol{T}=-\sigma \nabla \cdot(\nabla C /\|\nabla C\|) \nabla C$, where $\sigma$ denotes the surface tension and $C$ equals $C_{1}$ or $C_{2}$. A direct extension of this formulation involving suitable linear combinations of the three interfacial tensions was recently proposed by Kim \& Lowengrub (2005) and Kim (2007) in the context of a phase-field approach. We use expression (3.5) because, with the proper summation, it applies directly to an arbitrary number of phases, in contrast with the aforementioned formulation which is specific to three-phase systems (a closely related, albeit somewhat more complex formulation valid in an arbitrary number of phases has been proposed by Kim (2009), again in the context of a phase-field approach).

To establish (3.5) it is first worth noting that, having defined $C_{i j}=C_{i} /\left(C_{i}+C_{j}\right)=$ $1-C_{j i}$, we have $\left(C_{i}+C_{j}\right)^{2} \nabla C_{i j}=C_{j} \nabla C_{i}-C_{i} \nabla C_{j}$. Then, let us suppose that in a one-dimensional system the spatial distribution of the fluid phases is such that phase 1 (respectively 3) fills the interval $-\infty<x<x_{12}$ (respectively $x_{23}<x<+\infty$ ) while phase 2 fills the gap $x_{12}<x<x_{23}$. If interfaces are infinitely thin, we then have $C_{1}=\mathrm{H}\left(x_{12}-x\right), C_{3}=\mathrm{H}\left(x-x_{23}\right), C_{2}=\mathrm{H}\left(x-x_{12}\right) \mathrm{H}\left(x_{23}-x\right)$, where $\mathrm{H}$ denotes the Heaviside function. Hence

$$
\left.\begin{array}{rl}
\left(C_{i}+\right. & \left.C_{j}\right)^{2}\left\|\nabla C_{i j}\right\|= \\
& 0 \text { for } i=j \\
& 0 \quad \text { for } i=1, j=3 \text { or } i=3, j=1 \\
& \delta\left(x-x_{12}\right) \quad \text { for } i=1, j=2 \text { or } i=2, j=1 \\
& \delta\left(x-x_{23}\right) \quad \text { for } i=2, j=3 \text { or } i=3, j=2,
\end{array}\right\}
$$

where $\delta$ is the Dirac function. An important feature in (A 1) is that $\left\|\nabla C_{i j}\right\|$ is non-zero only if phases $i$ and $j$ have a common interface, as it should be. Now, at such 
an interface, $\nabla C_{i}=-\nabla C_{j}$, so $\nabla C_{i j}=\nabla C_{i}$ is aligned with the local normal to the interface. Hence one may define the unit normal pointing from phase $i$ toward phase $j$ as

$$
\boldsymbol{n}_{i j}=\nabla C_{i j} /\left\|\nabla C_{i j}\right\| .
$$

Generalizing (A 1) to three dimensions and using (A 2), (3.5) is found to reduce to

$$
\boldsymbol{T}=-\sum_{i=1}^{3} \sum_{j>i} \sigma_{i j}\left(\nabla \cdot \boldsymbol{n}_{i j}\right) \boldsymbol{n}_{i j} \delta_{A i j},
$$

where $\delta_{A i j}$ is the surface Dirac function which is non-zero only at the interface of area $A_{i j}$ between phases $i$ and $j$ and satisfies the normalization condition $\int_{V} \delta_{A i j} \mathrm{~d} V=A_{i j}$. Hence (3.5) is just the sum of the individual contributions of the three pairs of fluids $(i, j)$ with $i \neq j$ to the capillary force density in a three-phase system.

\section{Appendix B. Numerical volume conservation in a three-phase system}

As in Bonometti \& Magnaudet (2006, 2007), the JADIM code solves (3.1) by means of a direction-splitted version of Zalesak's flux corrected transport algorithm (Zalesak 1979). As most schemes used in the treatment of hyperbolic equations, this algorithm is not strictly conservative. This results in small fluctuations of the total volume filled by each fluid which may accumulate in time and lead to unacceptable volume variations over long times. An overall procedure maintaining the total volume variation of each phase within prescribed bounds was devised by Bonometti \& Magnaudet (2007). In a two-phase flow it is customary to solve (3.1) only in one of the fluids, say fluid 1, and make use of the relation $C_{1}+C_{2}=1$ to infer the local volume fraction of fluid 2. One may apply a similar procedure to three-phase flows by solving (3.1) in fluids 1 and 2 for instance and infer $C_{3}$ through $C_{3}=1-C_{1}-C_{2}$. However, due to the lack of strict conservativity of the algorithm, it may be that the numerical solution of (3.1) yields locally $C_{1}+C_{2}=1-\epsilon_{c}$ with $0<\epsilon_{c} \ll 1$, although only fluids 1 and 2 are actually present at that location or even in the entire flow domain. In such a case, a non-zero volume fraction of fluid $3, C_{3}=\epsilon_{c}$, spuriously appears (for instance droplets of light fluid may appear in the near-wake of the bubble when it rises in the heavy fluid!). To remove this artifact we solve (3.1) in all three fluid phases. Although this procedure slightly increases the overall computational cost, it has the decisive advantage that all phases are treated in a similar manner and that none of them is created artificially by the cumulative effect of errors made in the transport of the other two. The drawback of this procedure is that, since the algorithm does not ensure a strict volume conservation of each phase, none of the three volume fraction fields it produces, say $C_{i}^{*}$, guarantees this conservation, and the relation $C_{1}^{*}+C_{2}^{*}+C_{3}^{*}=1$ is not satisfied. We deal with these issues as follows. First we apply the overall volume conservation correction devised by Bonometti \& Magnaudet (2007) to the gas that fills the bubble, say phase 2 , which yields the corrected volume fraction field $C_{2} \approx C_{2}^{*}$. The reason for privileging conservation of the bubble volume is that this parameter is of primary importance in the present problem, as it determines the buoyancy force that drives the entire system. In contrast, both liquids are almost stagnant except in the region crossed by the bubble, so that minute departures from volume conservation in phases 1 and 3 have strictly no effect on the dynamics of the system. Finally, to enforce the overall conservation property $C_{1}+C_{2}+C_{3}=1$, we renormalize any local 
(a)

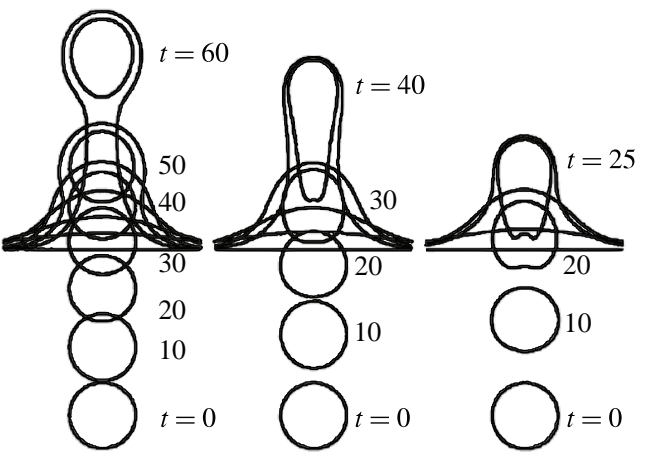

(b)

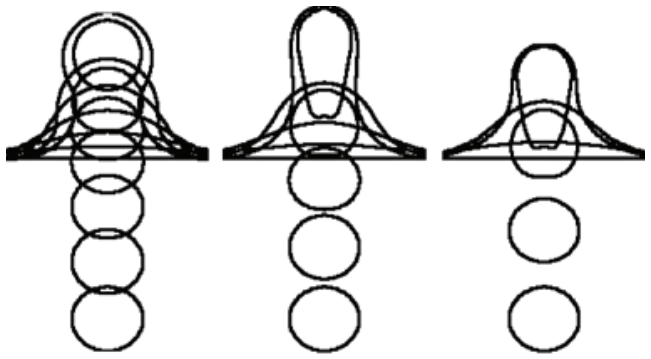

FIGURE 17. Evolution of the shape and position of the drop in the BIM computations of (a) MS and (b) JADIM for $\left(\rho_{1}-\rho_{3}\right) /\left(\rho_{1}-\rho_{2}\right)=0.2,\left(\rho_{1}-\rho_{2}\right) g d^{2} / \sigma_{12}=80, \mu_{3} / \mu_{1}=0.1$, $\sigma_{13} / \sigma_{12}=0$, and three different drop/lower liquid viscosity ratios: $\lambda=10$ (left), 1 (middle) and 0.1 (right). Times are normalized with the viscous time scale $2 \mu_{1} /\left(\rho_{1}-\rho_{2}\right) g d$.

value of the volume fraction produced by the direction-splitted Zalesak algorithm in the two liquid phases in the form $C_{\alpha}=C_{\alpha}^{*}\left(1-C_{2}\right) /\left(C_{1}^{*}+C_{3}^{*}\right)$, with $\alpha=1$ or 3 .

\section{Appendix C. Comparison with predictions of a BIM approach}

To check the accuracy of our code in a configuration close to those of interest here, we reconsider some of the low-Reynolds-number flows computed by Manga \& Stone (1995) (hereinafter referred to as MS) using a BIM. Initially spherical drops (whose inner fluid is referred to as fluid 2) with a diameter $d$ are released from rest within fluid 1 with their top located at a position $h / d=-2$ with respect to the flat horizontal interface separating fluids 1 and 3 . Then they rise under the effect of gravity until they come close to that interface. Grid characteristics are similar to those mentioned in $\S 3$. Computations are run over the same amount of dimensionless time $T=t\left(\rho_{1}-\rho_{2}\right) g d /\left(2 \mu_{1}\right)$ as in MS, whose results we take as a reference even though their exact accuracy is unknown.

The position and shape of the various interfaces at selected values of $T$ are displayed in figure 17 for three values of the viscosity ratio $\lambda=\mu_{2} / \mu_{1}$, namely $\lambda=0.1,1$ and 10 . The results obtained with the two codes compare well for the lowest value of $\lambda$. The final shape and position of the drop predicted by JADIM are very close to those reported by MS. For the other two values of $\lambda$, although satisfactory until the entire drop has crossed the initial position of the interface between fluids 1 and 3 (say $h / d \approx 1$ ), the agreement deteriorates at later times. The drop deformation then becomes underpredicted (see in particular the tail of the final drop in the intermediate case $\lambda=1$ ) and so is the final altitude reached by its top; 

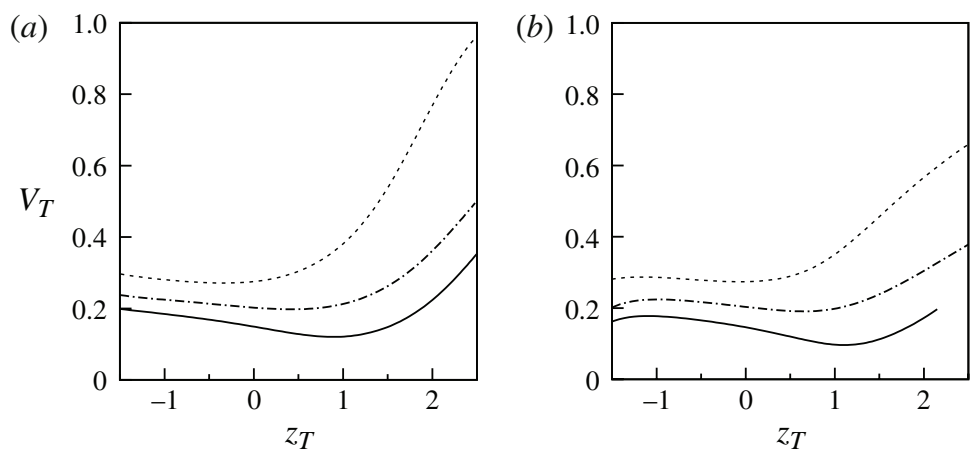

FIGURE 18. Evolution of the rise speed of the top of the drop in $(a)$ the BIM computations of MS and $(b)$ JADIM. Solid line, $\lambda=10$; dot-dashed line, $\lambda=1$; dashed line, $\lambda=0.1$. Dimensionless parameters are similar to those of figure 17. Velocities are normalized with the viscous scale $\left(\rho_{1}-\rho_{2}\right) g d^{2} / 4 \mu_{1}$; positions are normalized with the initial drop diameter $d$ and refer to the top of the drop relative to the initial position of the interface between fluids 1 and 3 .
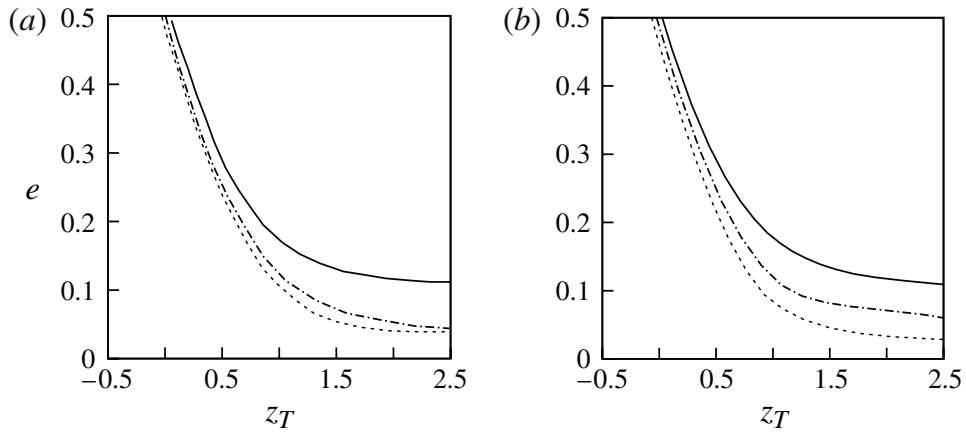

FIGURE 19. Evolution of the gap thickness (normalized with $d$ ) in $(a)$ the BIM computations of MS and (b) JADIM. Dimensionless parameters are similar to those of figure 17; symbols are similar to those of figure 18.

the larger $\lambda$, the larger the difference in this altitude. The normalized velocity $V_{T}$ of the top of the drop (defined as $V_{T}=4 V \mu_{1} /\left(\rho_{1}-\rho_{2}\right) g d^{2}$ ) is plotted as a function of the current normalized position $z_{T}$ of that uppermost point in figure 18. As seen in this figure, after the drop has reached a rise speed close to that predicted by the Hadamard-Rybczyinski theory $\left(V_{T}=2(1+\lambda) / 3(2+3 \lambda)\right)$, it first slows down until its top reaches a certain position $z_{T c}$ located above the initial position of the interface between fluids 1 and 3 . Since the larger $\lambda$ the slower the drop deformation, the location of $z_{T c}$ increases with $\lambda$, ranging from $z_{T c} \approx 0.25$ for $\lambda=0.1$ to $z_{T c} \approx 1.25$ for $\lambda=10$. This trend is well captured by our code. The situation deteriorates at a later time, when the rise speed experiences a sharp increase. The code predicts this acceleration but its magnitude is significantly less than that found with the BIM. Figure 19 shows how the normalized thickness $e$ of the film of fluid 1 on top of the drop evolves. The initial sharp decrease of $e$ is followed by a much slower decrease corresponding to the drainage of the film. This second stage is fairly well captured by 


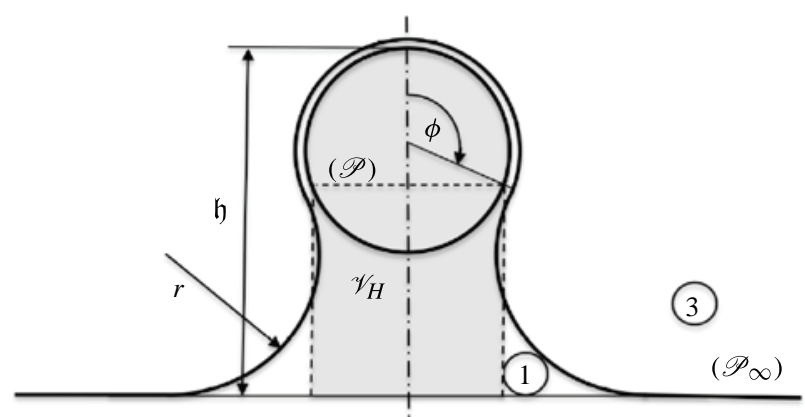

FIGURE 20. Sketch of the film + tail model.

our code, although the drainage appears to be somewhat too slow or too fast for $\lambda=1$ and $\lambda=0.1$, respectively.

Differences observed between the predictions of our code, in which gradients of $C_{i}$ are spread over interfacial regions with a small but finite thickness, and those of the BIM, in which interfaces are sharp, may readily be explained. Indeed, the finite thickness of interfacial regions results in a spreading of both the capillary force (through its dependence on $\nabla C_{i}$ ) and the viscosity variations (through the dependence of viscosity on $C_{i}$ ) within the film. When the interface between fluids 1 and 2 comes close to that between fluids 1 and 3 , the latter interface, which in the present computations has no interfacial tension, starts to experience some artificial capillary force. Hence it resists deformation more than it should, thus delaying the rise of the drop and hindering its deformation. Depending on the values of $\mu_{2}$ and $\mu_{3}$, the average viscosity in the gap is somewhat smaller or larger than its actual value $\mu_{1}$, so it takes less or more time than it should for the film to be drained. The above two effects should cooperate and result in a slower rise and deformation of the drop when $\lambda>1$, while they should counteract each other when $\lambda<1$. Based on this reasoning, the predictions of our code for a given $\mu_{3}$ are expected to be more accurate with bubbles $(\lambda \ll 1)$ than with very viscous drops $(\lambda \gg 1)$. The results displayed in figures 17 and 18 fully support these views. Significant differences between predictions of the present code and BIM results are seen to start typically for $e \lesssim 0.04$.

\section{Appendix D. A static model of the film-drainage/tailing transition}

Let us consider a spherical bubble of diameter $d$ at the time when its top stands at an altitude $z=h$ above the plane $\left(\mathscr{P}_{\infty}\right)$ corresponding to the undisturbed position of the fluid-fluid interface and part of its surface is covered by a thin film (see figure 20), for example that part between its upward axis (which we define as $\theta=0$ ) and a certain cap angle $\theta=\phi$. We assume that the film thickens rapidly at larger angular positions, so it has no direct effect on the bubble for $\theta>\phi$ (the first four snapshots of the experimental sequence in figure 6(a) provide a clear illustration of that situation). If dynamical effects in the upper fluid and pressure variations across the film are negligibly small, the pressure on the part of the bubble surface corresponding to $\theta<\phi$ is $-\rho_{3} g z+4 \sigma_{13} / d$. Similarly, provided dynamical effects in the vicinity of the bubble are negligible in the heavy fluid, the corresponding pressure for $\theta>\phi$ is $-\rho_{1} g z$.

Setting $\mathfrak{h}=h / d$ and integrating the pressure distribution over the bubble surface, simple geometrical considerations show that the net buoyancy force on the bubble 
is $\rho_{1} g \mathscr{V}\left(1-R \mathscr{V}_{H}(\phi)-6 B_{I}^{-1} \sin ^{2} \phi\right)$, where $\mathscr{V}_{H}(\phi)=(3 / 2) \mathfrak{h}(\phi) \sin ^{2} \phi-(1 / 4)\left(1-3 \cos ^{2} \phi+\right.$ $2 \cos ^{3} \phi$ ) is the dimensionless volume corresponding to the grey region in figure 20 ,

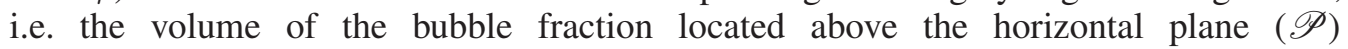
corresponding to $\theta=\phi$ plus that of the vertical cylinder of dimensionless diameter $\sin \phi$ bounded by planes $(\mathscr{P})$ and $\left(\mathscr{P}_{\infty}\right)$. In other words, the density difference between the two fluids and the capillary force at their interface reduce the local effective driving force per unit mass acting on the bubble to

$$
g_{e}(\phi)=g\left\{1-R\left[\frac{3}{2} \mathfrak{h}(\phi) \sin ^{2} \phi-\frac{1}{4}\left(1-3 \cos ^{2} \phi+2 \cos ^{3} \phi\right)\right]-\frac{6}{B_{I}} \sin ^{2} \phi\right\} .
$$

Determining how $\mathfrak{h}$ varies with $\phi$ requires the shape of the column to be known, and this shape is governed by the force balance in the corresponding flow region. A simple, although crude, procedure to achieve this determination (sketched in figure 20) was followed by Maru et al. (1971) who assumed the vertical crosssection of the column to be bounded by an arc of a circle tangent to the film at $\theta=\phi$ as well as at the point where the column matches the undisturbed interface. Requesting that Laplace's law be satisfied at the intersection of the bubble surface with $(\mathscr{P})$ then implies $\left(\rho_{1}-\rho_{3}\right) g z(\phi)=-\sigma_{13} \kappa(\phi)$, where $\kappa$ is twice the local mean curvature of the column surface. Simple algebra finally allows the dimensionless arc radius $\mathfrak{r}$ to be calculated, yielding $\mathfrak{h}(\phi)=(\mathfrak{r}+1 / 2)(1-\cos \phi)$ with $\mathfrak{r}=\left\{-1+\left[1+R B_{I}(1-\cos \phi)\right]^{1 / 2}\right\} /\left\{R B_{I}(1-\cos \phi)\right\}$.

In the limit of small $R B_{I}$, this expression tends toward $\mathfrak{h}(\phi)=1-\cos \phi+O\left(R B_{I}\right)$, which yields $\mathfrak{h}(\phi=\pi / 2) \approx 1$, suggesting that the upper half of the bubble is almost entirely covered by the film when its bottom stands on $\left(\mathscr{P}_{\infty}\right)$. This is indeed approximately what is observed here with the spheroidal bubbles of figures 4 and 6 . Hence we regard the above expression for $\mathfrak{h}$ as sufficient given the limited scope of the present model, although it is certainly unrealistic at large $\phi$ since $\mathfrak{h}$ actually goes to infinity as $\phi \rightarrow \pi$. Assuming that it is still valid in the range of $\phi$ where, given the condition $\mathscr{V}_{H} \rightarrow 1$ for $\phi \rightarrow \pi$, we expect $\mathscr{V}_{H}$ to pass through a maximum, this model predicts this maximum to occur at $\phi=2 \pi / 3$, where $\mathscr{V}_{H} \approx 27 / 16$. Hence $g_{e}$ reaches its minimum, $g_{m}$, at some angle $\phi_{m}$ in the range ] $\pi / 2,2 \pi / 3\left[\right.$ such that $\cos \phi_{m} \approx-R B_{I} / 8$, so that

$$
g_{m} \approx g\left\{1-\frac{5}{4} R\left[1+\frac{3}{20} R B_{I}\right]-6 B_{I}^{-1}\right\} .
$$

In the opposite limit of large $R B_{I}$, assuming that capillary effects, although small, still control the shape of the column, the above model predicts $\mathfrak{r} \rightarrow$ $\left(R B_{I}\right)^{-1 / 2}(1-\cos \phi)^{-1 / 2}$ and $\mathfrak{h} \rightarrow(1-\cos \phi) / 2+\left(R B_{I}\right)^{-1 / 2}(1-\cos \phi)^{1 / 2}$. Now $\mathscr{V}_{H}$ (respectively $g_{m}$ ) reaches its maximum (respectively minimum) for $\phi \approx \pi-$ $2\left(R B_{I} / 2\right)^{-1 / 4}$, where $\mathscr{V}_{H} \approx 1+6\left(R B_{I}\right)^{-1}$, so that

$$
g_{m} \approx g\left\{1-R-6 B_{I}^{-1}\right\} .
$$

Note that, according to (D 3), $g_{m}$ keeps a positive value in systems with a large interfacial Bond number only if $B_{I}>6 /(1-R)$, which is simply the criterion derived by Greene et al. (1988).

It must be stressed that the above model only takes into account static effects (gravity and interfacial tension) and neglects any influence of viscous and inertial mechanisms. This is a clear limitation since Geller et al. (1986) showed that, under Stokes flow conditions, a rigid sphere approaching a fluid-fluid interface may, for certain sets of $R$ and $B_{I}$, give birth to either a film-drainage or a tailing configuration, 
depending on whether $\Lambda$ is large or small. The most questionable aspect of the above model (as well as of that of Maru et al. (1971) which involves the same ingredients) is certainly the closure we use to relate $\mathfrak{h}$ to $\phi$, especially because it makes the column geometry entirely dependent on capillary effects whereas it is well established that the same type of structure may exist with miscible fluids (Srdić-Mitrović et al. 1999; Camassa et al. 2009). Our belief, reinforced by the comparison with experimental results, is that this closure is approximately valid as far as the column is 'short', say $\mathfrak{h} \leqslant 3 / 2$, which corresponds to $\phi \leqslant 2 \pi / 3$, because the gap between the region where its shape is controlled by the matching with the film at $\theta=\phi$ and that where it is controlled by the matching with the undisturbed interface is small. For larger gaps or nearly miscible fluids $\left(B_{I} \rightarrow \infty\right)$, there is no doubt that dynamical effects have to be taken into account to predict $\mathfrak{h}$ realistically (see e.g. the recent low-Reynolds-number investigation by Lee \& Kim 2011). However, this is clearly beyond the scope of the present analysis, whose goal is only to show qualitatively why increasing $B_{I}$ makes the bubble cross the initial position of the interface easier. Based on these remarks, we regard the $g_{m}>0$ condition provided by (D2) or (D 3) as a necessary condition for the tailing configuration to occur, but there is little doubt that the film-drainage configuration may emerge for positive $g_{m}$ if $\Lambda$ is large enough.

\section{REFERENCES}

Allan, R. S., Charles, G. E. \& MAson, S. G. 1961 The approach of gas bubbles to a gas/liquid interface. J. Colloid Sci. 16, 150-165.

BART, E. 1968 The slow unsteady settling of a fluid sphere toward a flat fluid interface. Chem. Engng Sci. 23, 193-210.

Bonometti, T. \& Magnaudet, J. 2006 Transition from spherical caps to toroidal bubbles. Phys. Fluids 18, 052102.

Bonometti, T. \& Magnaudet, J. 2007 An interface-capturing method for incompressible two-phase flows: validation and application to bubble dynamics. Intl J. Multiphase Flow 33, 109-133.

Brackbill, J. U., Kothe, D. B. \& Zemach, C. 1992 A continuum method for modelling surface tension. J. Comput. Phys. 100, 335-354.

Bush, J. W. M. \& EAmeS, I. 1998 Fluid displacement by high Reynolds number bubble motion in a thin gap. Intl J. Multiphase Flow 24, 411-430.

Camassa, R., Falcon, C., Lin, J., Mclaughlin, R. M. \& Parker, R. 2009 Prolonged residence times for particles settling through stratified miscible fluids in the Stokes regime. Phys. Fluids 21, 031702.

Charles, G. E. \& Mason, S. G. 1960 The coalescence of liquid drops with flat liquid/liquid interfaces. J. Colloid Sci. 15, 236-267.

CHI, B. K. \& LEAL, L. G. 1989 A theoretical study of the motion of a viscous drop toward a planar wall at low Reynolds number. J. Fluid Mech. 201, 123-146.

Cranga, J., Gardin, P., Huin, D. \& Magnaudet, J. 2001 Influence of surface pressure and slag layer on bubble bursting in degasser systems. In Computational Modelling of Materials, Minerals, and Metals Processing (ed. M. Cross, J. W. Evans \& C. Bailey), pp. 105-114. The Minerals, Metals \& Materials Society.

DAVIES, R. M. \& TAYLOR, G. I. 1950 The mechanics of large bubbles rising through liquids in tubes. Proc. R. Soc. Lond. A 200, 375-390.

Debrégeas, G., De Gennes, P.-G. \& Brochard-Wyart, F. 1998 The life and death of 'bare' viscous bubbles. Science 279, 1704-1707.

Dietrich, N., Poncin, S., Pheulpin, S. \& Li, H. Z. 2008 Passage of a bubble through a liquid-liquid interface. AIChE J. 54, 594-600.

EAmes, I. \& DuURsma, G. 1997 Displacement of horizontal layers by bubbles injected into fluidized beds. Chem. Engng Sci. 52, 2697-2705. 
Geller, A. S., Lee, S. H. \& Leal, L. G. 1986 The creeping motion of a spherical particle normal to a deformable interface. J. Fluid Mech. 169, 27-69.

De Gennes, P.-G., Brochard-Wyart, F. \& Quéré, D. 2004 Capillarity and Wetting Phenomena: Drops, Bubbles, Pearls and Waves. Springer.

Greene, G. A., Chen, J. C. \& Conlin, M. T. 1988 Onset of entrainment between immiscible liquid layers due to rising gas bubbles. Intl J. Heat Mass Transfer 31, 1309-1317.

Greene, G. A., Chen, J. C. \& Conlin, M. T. 1991 Bubble induced entrainment between stratified liquid layers. Intl J. Heat Mass Transfer 34, 149-157.

HARTLAND, S. 1968 The approach of a rigid sphere to a deformable liquid/liquid interface. J. Colloid Interface Sci. 26, 383-394.

HARTLAND, S. 1969 The profile of the draining film between a rigid sphere and a deformable fluid-liquid interface. Chem. Engng Sci. 24, 987-995.

Jones, A. F. \& Wilson, S. D. R. 1978 The film drainage problem in droplet coalescence. J. Fluid Mech. 287, 279-298.

Joseph, D. D. 2003 Rise velocity of a spherical cap bubble. J. Fluid Mech. 488, 213-223.

Kemina, M., Olmos, E., FeI, W., Poncin, S. \& Li, H. Z. 2007 Passage of a gas bubble through a liquid-liquid interface. Ind. Engng Chem. Res. 46, 6099-6104.

KIM, J. 2007 Phase field computations for ternary fluid flows. Comput. Meth. Appl. Mech. Engng 196, 4779-4788.

KIM, J. 2009 A generalized continuous surface tension force formulation for phase-field models for multi-component immiscible fluid flows. Comput. Meth. Appl. Mech. Engng 198, 3105-3112.

Kim, J. \& Lowengrub, J. 2005 Phase field modelling and simulation of three-phase flows. Interface Free Bound. 7, 435-466.

KobayAshi, S. 1993 Iron droplet formation due to bubbles passing through molten iron/slag interface. ISIJ Int. 33, 577-582.

LEAL, L. G. \& LEE, S. H. 1982 Particle motion near a deformable fluid interface. Adv. Colloid Interface Sci. 17, 61-81.

LEE, D. G. \& Kim, H. Y. 2011 Sinking of small sphere at low Reynolds number through interface. Phys. Fluids 23, 072104.

Lundgren, T. S. \& Mansour, N. N. 1991 Vortex ring bubbles. J. Fluid Mech. 87, 263-288.

Magnaudet, J. \& Mougin, G. 2007 Wake instability of a fixed spheroidal bubble. J. Fluid Mech. 572, 311-337.

MangA, M. \& Stone, H. A. 1995 Low Reynolds number motion of bubbles, drops and rigid spheres through fluid-fluid interfaces. J. Fluid Mech. 287, 279-298.

Manga, M., Stone, H. A. \& O'Connell, R. L. 1993 The interaction of plume heads with compositional discontinuities in the Earth's mantle. J. Geophys. Res. 98, 19979-19990.

MARU, H. C., Wasan, D. T. \& Kintner, R. C. 1971 Behavior of a rigid sphere at a liquid-liquid interface. Chem. Engng Sci. 26, 1615-1628.

MeIron, D. I. 1989 On the stability of gas bubbles rising in an inviscid fluid. J. Fluid Mech. 198, 101-114.

Miksis, M., Vanden-Broeck, J. M. \& Keller, J. B. 1981 Axisymmetric bubble or drop in a uniform flow. J. Fluid Mech. 108, 89-100.

Mohamed-Kassim, Z. \& Longmire, E. K. 2004 Drop coalescence through a liquid-liquid interface. Phys. Fluids 16, 2170-2181.

Moore, D. W. 1963 The boundary layer on a spherical gas bubble. J. Fluid Mech. 16, 161-176.

Moore, D. W. 1965 The velocity of rise of distorted gas bubbles in a liquid of small viscosity. J. Fluid Mech. 23, 749-766.

NATARAJAN, R. \& ACRIVOS, A. 1993 The instability of the steady flow past spheres and disks. J. Fluid Mech. 254, 323-344.

Pedley, T. J. 1968 The toroidal bubble. J. Fluid Mech. 32, 97-112.

Pigeonneau, F. \& Sellier, A. 2011 Low-Reynolds-number gravity-driven migration and deformation of bubbles near a free surface. Phys. Fluids 23, 092102.

Poggi, D., Minto, M. \& Davenport, W. G. 1969 Mechanisms of metal entrapment in slags. J. Met. 21, 40-45. 
Popinet, S. 2003 Gerris: a tree-based adaptive solver for the incompressible Euler equations in complex geometries. J. Comput. Phys. 190, 572-600.

Popinet, S. 2009 An accurate adaptive solver for surface-tension-driven interfacial flows. J. Comput. Phys. 228, 5838-5866.

PrinCEn, H. M. 1963 Shape of a fluid drop at a liquid-liquid interface. J. Colloid Sci. 18, $178-195$.

Princen, H. M. \& MASON, S. G. $1965 a$ Shape of a fluid drop at a fluid-liquid interface. Part 1. Extension and test of two-phase theory. J. Colloid Sci. 20, 156-172.

Princen, H. M. \& Mason, S. G. 1965b Shape of a fluid drop at a fluid-liquid interface. Part 2. Theory for three-phase systems. J. Colloid Sci. 20, 246-266.

Reiter, G. \& Schwerdtfeger, K. $1992 a$ Observation of physical phenomena occurring during passage of bubbles through liquid/liquid interfaces. ISIJ Int. 32, 50-56.

Reiter, G. \& Schwerdtfeger, K. 1992b Characteristics of entrainment at liquid/liquid interfaces due to rising bubbles. ISIJ Int. 32, 57-65.

Shah, S. T., Wasan, D. T. \& Kintner, R. C. 1972 Passage of a liquid drop through a liquid-liquid interface. Chem. Engng Sci. 27, 881-893.

Shopov, P. J. \& MineV, P. D. 1992 The unsteady motion of a bubble or drop towards a liquid-liquid interface. J. Fluid Mech. 235, 123-141.

Smith, P. G. \& VAN DE Ven, T. G. M. 1984 The effect of gravity on the drainage of a thin liquid film between a solid sphere and a liquid/fluid interface. J. Colloid Interface Sci. 100, 456-464.

Srdić-Mitrović, A. N., Mohamed, N. A. \& Fernando, H. J. S. 1999 Gravitational settling of particles through density interfaces. J. Fluid Mech. 381, 175-198.

TAYLOR, T. D. \& ACRivos, A. 1964 On the deformation and drag of a falling viscous drop at low Reynolds number. J. Fluid Mech. 18, 466-476.

Thomas, S., Esmaeeli, A. \& Tryggvason, G. 2010 Multiscale computations of thin films in multiphase flows. Intl J. Multiphase Flow 36, 71-77.

Tsai, S. S. H., Wexler, J. S., Wan, J. \& Stone, H. A. 2011 Conformal coating of particles in microchannels by magnetic forcing. Appl. Phys. Lett. 99, 153509.

Walters, J. K. \& DaVidson, J. F. 1963 The initial motion of a gas bubble formed in a inviscid liquid. Part 2. The three-dimensional bubble and the toroidal bubble. J. Fluid Mech. 17, 321-336.

ZalesaK, S. T. 1979 Fully multidimensional Flux-Corrected Transport algorithms for fluids. J. Comput. Phys. 31, 335-362. 